\title{
EINLEITUNG
}

\author{
Jeder Mensch lebt zwei Leben: \\ sein eigenes und das seiner Zeitgenossen.
}

Thomas Mann

Nachdem Anna d'Este im Mai 1607 gestorben war, erschien in Frankreich eine ganze Reihe von Lobgedichten, Leichenpredigten und anderen Funeralwerken, in denen das Wesen der italienischen Prinzessin besungen und an die Stationen ihres Lebens erinnert wurde. In einem dieser Gedichte treffen die Götter im Kinderzimmer der Neugeborenen zusammen, um sie mit ihren Gaben zu segnen. Mars und Venus treten gemeinsam an die Wiege:

D'un courage eslevé Mars luy enfla le cceur,

La purgeat si bien de ce timide humeur,

Que coustumierement sur la femme domine,

Qu'en genereux dessains il la fit masculine.

La courtoise Venus fit aussi tant d'effortz,

Pour, des rares beautés, luy decorer le corps,

Que paravant n'apres d'en voir une si belle,

C'est chose qu'on ne peut, non pas mesme pareille'.

Diese wenigen Zeilen verdeutlichen bereits die Zwiespältigkeit, mit der die Zeitgenossen die Herzogin sahen: So mutig war sie, daß sie masculine erschien, während sich ihr Körper mit seltener Schönheit geschmückt präsentierte. Daß solch unterschiedliche Sichtweisen auch in Darstellungen und Selbstdarstellungen anderer Fürstinnen $\mathrm{zu}$ finden sind, zeigen Margarete von Navarra, der in Lobgesăngen die Natur einer Frau und die Vortrefflichkeit eines Mannes, ein Frauenkörper und ein Männerherz zugeschrieben wurden, und Sophie von Hannover, die ihre eigene Person in Briefen und Memoiren teils als einflußreich und mächtig, teils als hilflos und ohnmächtig beschrieb ${ }^{2}$. Diese Beispiele und die zitierten Zeilen uber die Herzogin von Nemours verdeutlichen, welchen Fragestellungen sich die Biographie einer adligen Dame daher sinnvollerweise widmen sollte. Und so beschäftigt sich diese Arbeit uber Anna d'Este, Tochter des Herzogs von Ferrara und der Renée de France,

' LA PALUD, Discour funebre sur la mort de tres-Illustre Princesse Anne D'Est Duchesse de Genevois, Nemours, Chartres, etc., Chambéry o.J. [1609], S. 8f.

${ }^{2}$ Margarete ZiMmermanN, Salon der Autorinnen. Französische dames de lettres vom Mittelalter bis zum 17. Jahrhundert, Berlin 2005, S. 127; Ute DANIEL, Zwischen Zentrum und Peripherie der Hofgesellschaft: Zur biographischen Struktur eines Fürstinnenlebens der Frlhen Neuzeit am Beispiel der Kurfürstin Sophie von Hannover, in: L'Homme 8 (1997) S. 208-217. Vgl. den Hinweis auf das Lob der Christine de Pizan als "virilis femina", in: Patricia H. LABALME, Einleitung zu: DIES. (Hg.), Beyond Their Sex. Learned Women of the European Past, New York, London 1980, S. 1-8, hier S. 5. 
in erster Ehe Herzogin von Aumale und von Guise, in zweiter Herzogin von Nemours, auch eher mit Darstellungen und Wahrnehmungen als mit der Suche nach dem $>$ Wie-sie-wirklich-war .

\section{Forschungsstand und Quellenlage}

Zu Beginn der 1920er Jahre wurden in Frankreich kurz hintereinander zwei Schriften veröffentlicht, die sich mit Anna d'Este beschäftigen. Schon an den Titeln ist zu erkennen, welche Fragestellungen diese Bücher verfolgen. In ihrer "Véritable Princesse de Clèves« vertritt Valentine Poizat die These, Madame de La Fayette habe die historische Anna vor Augen gehabt, als sie die Figur der Mademoiselle de Chartres, der späteren Prinzessin von Clèves, fur ihren gleichnamigen Roman entwarf; als Beweis werden Ausschnitte aus den Quellentexten den jeweils korrespondierenden Stellen des Romans gegenubergestellt ${ }^{3}$. So zweifelhaft dieses Vorgehen auch sein mag, der Autorin kommt das Verdienst zu, die Handschriftenabteilung der Pariser Nationalbibliothek nach Dokumenten über die italienische Prinzessin durchsucht zu haben. Als Reaktion auf dieses Buch erschien kurze Zeit später, zunăchst in der »Revue des deux mondes «, dann als eigene Veröffentlichung, »Les amants d'Annecy « von Henry Bordeaux. Vehement widerspricht der Romancier der Meinung seiner Kollegin, Anna habe, parallel zu Mademoiselle de Chartres, schon während ihrer ersten Ehe eine Neigung zum Herzog von Nemours verspürt. Der Geschichte um die Prinzessin, ihren Gemahl und den vermeintlichen Liebhaber fülte sich der Autor besonders verbunden, was einer der Gründe dafur ist, daß er Annas angeblich beschädigten Ruf einer whonnête femme« $\mathrm{zu}$ retablieren suchte ${ }^{4}$.

Man mag die Konstruktionen dieser Bücher belächeln, und tatsächlich fehlt beiden der wissenschaftliche Anspruch völlig, doch stellen sie bis heute die einzigen einigermaßen umfassenden Abhandlungen zu Anna d'Este dar. Und das, obwohl die Tochter von Renée de France und Ercole d'Este in den Korrespondenzen und Memoiren ihrer Zeitgenossen einen bedeutenden Platz einnimmt. Bei Pierre de Bourdeille, Seigneur de Brantôme, ist sie oft präsent, die Anmerkungen über die Stadt Paris des Pierre de L'Estoile wären nur halb so interessant, würde nicht an vielen Stellen über die Taten der italienischen

\footnotetext{
${ }^{3}$ Valentine PoIZAT, La Véritable Princesse de Clèves, Paris 1920.

${ }^{4}$ Das Zitat stammt aus der Rezension von Gustave Baguenault de Puchesse zu dem in der "Revue des deux mondes" erstmals veroffentlichten Artikel, wieder abgedruckt im Anhang zu: Henry BORDEAUX, Les amants d'Annecy. Anne d'Este et Jacques de Nemours, Paris, Chambéry 1921, S. 97-99, hier S. 98.
} 
Prinzessin berichtet, Jacques-Auguste de Thou widmete ihr ganze Seiten in seinem Geschichtswerk, in der Korrespondenz des Savoyer Gesandten am französischen Hof, René de Lucinge, taucht ihr Name zu manchen Zeiten ebenso häufig auf wie derjenige der Königinmutter, schließlich haben Pierre de Ronsard und Torquato Tasso der Herzogin ihre Verse gewidmet. Hinzu kommt, daß bereits dreizehn Jahre nach Annas Tod eine erste biographische Skizze verfugbar war, veröffentlicht von Francesco Agostino della Chiesa in seinem "Theatro delle donne letterate«, wo unter dem merkwilirdigen Stichwort "Anna di Savoia《 an das Engagement der Herzogin non solo col sovenir a bisogni, ma con la lingua, e col conseglio während der Belagerung von Paris durch die Truppen Heinrichs IV. erinnert wird. Zehn Jahre später erschien eine ihr gewidmete Lebensbeschreibung in den "Éloges et vies des reynes, princesses, dames et damoiselles illustres « des Hilarion de Coste, auf die wiederum Oudin in seiner Manuskript gebliebenen Geschichte des Hauses Guise von 1647 zurückgriff, in welcher der Prinzessin ebenfalls ein überaus großer Stellenwert eingeräumt wird ${ }^{5}$.

Doch dann, nach della Chiesa, de Coste und Oudin, schweigen die Geschichtswerke zweihundert Jahre lang über diejenige, die einst eine der einflußreichsten Damen im Kreis um Katharina von Medici war, die mit ihren Prozessen den König selbst herauszufordern wagte, für zwei Ehegatten einen enorm großen Besitz verwaltete und sich für sieben ihrer zehn Kinder um Karrieren und Heiratsverbindungen kümmerte. Wie die anderen adligen Damen ihrer Zeit ist auch sie fast völlig verschwunden aus den Büchern und Erinnerungen, "gestrichen«, wie Éliane Viennot sich ausdrückt ${ }^{6}$. Und sie nennt ein sprechendes Beispiel: Während Mézeray in seiner Geschichte Frankreichs den Frauen aus dem Hause Guise noch den ihnen zustehenden Platz einrăumt, verschwinden sie aus dem von ihm selbst einige Zeit später besorgten Index zum Werk völlig. Hier werden etwa die Ereignisse in der Kirche von Meulan im September 1563 beschrieben als: les enfants du duc de Guise demandent justice au roy, während an der entsprechenden Stelle des Buches zu lesen ist, daß es nicht nur die Kinder, sondern auch die Witwe, Mutter und

\footnotetext{
${ }^{5}$ Francesco Agostino Della ChIESA, Art. "Di Anna di Savoia«, in: Ders., Theatro delle donne letterate, con un breve discorso della preminenza, e perfettione del sesso donnesco, Mondovì 1620, S. 70f.; Hilarion de CosTE, Anne d'Est ou de Ferare, Duchesse de Guise et de Nemours, in: DERS., Les éloges et vies des reynes, princesses, dames et damoiselles illustres, Paris 1630, S. 32-37; OUDIN, Histoire de la maison de Guise, 1647, in: BnF, Fr. 5798-5801.

${ }^{6}$ Éliane VIENNOT, Des »femmes d'État« au XVI siècle: les princesses de la Ligue et l'écriture de l'histoire, in: Danielle HAASE-DUBOSC, DIES. (Hg.), Femmes et pouvoirs sous l'ancien régime, Paris 1991, S. 77-97, hier S. 89: "En effet, ces femmes dont les faits et gestes avaient été consignés par beaucoup de témoins de la période ont été progressivement rayées de l'histoire.«
} 
Tanten waren, welche dem König ihr Anliegen auf derart einprägsame Weise vortrugen?.

Ab der Mitte des 19. Jahrhunderts findet sich Annas Name vereinzelt in biographischen Nachschlagewerken und Gesamtdarstellungen zu den französischen Religionskriegen. In der »Nouvelle biographie générale« nimmt der ihr gewidmete Artikel etwa eine Spalte ein, Novellis behandelt die Prinzessin in seinen "Donne celebri piemontesi«, und bei Michaud muß man lange suchen, da sie unter dem Stichwort $» F e r r a r e$, Anne de" aufgenommen ist ${ }^{8}$. Michelet vertritt zwar die Ansicht, die Herzogin sei nach dem Tod ihres ersten Gemahls wla vraie tête des Guises« gewesen, ignoriert sie aber ansonsten völlig in seiner Darstellung, von Anspielungen auf ihren "charme italien, et non pas peu du sang des Borgia abgesehen. In seinem 1873 erschienenen Werk kommt auch Édouard Frémy wiederholt und sehr ausfuhrlich auf die Prinzessin zu sprechen, sein Wissen uber sie schöpft er aber ausschließlich aus Brantôme und L'Estoile, nicht aus den im Untertitel des Buches versprochenen »documents nouveaux et inédits«. Ungedruckte Quellen verwendete hingegen Lucien Romier in seinen zwei Bänden über die »Origines politiques des guerres de religion «, in denen Anna, als Italienerin in Frankreich, und ihre Heirat mit dem Herzog von Aumale relativ ausfuhrlich, vor allem anhand von Dokumenten aus dem Staatsarchiv von Modena behandelt werden?.

Von Valentine Poizat und dem Lokalhistoriker von Annecy, ClaudeAntoine Ducis abgesehen, ist uberhaupt nie über Anna d'Este in Archiven und Handschriftenabteilungen der Bibliotheken geforscht worden, und bis heute beschränken sich alle Erwähnungen ihres Namens auf die immer gleichen Stellen in den Memoiren der Zeitgenossen. Das von Francesco Orestano besorgte Nachschlagewerk uber »Eroine, ispiratrici e donne di eccezione« aus dem Jahre 1940 zeigt, wie sich demnach das Leben der Herzogin von Guise und von Nemours zusammenfassen läßt: »nata nel 1531, figlia di Ercole II e di Renata di Francia, nel 1549 [!] ando sposa a Francesco, duca di Guisa, sopranominato lo Sfigurato. Mortole il primo marito, si rimaritò con Giacomo di Savoia, duca di Nemours. Dopo essere stata prigioniera nei castelli di Blois e

${ }^{7}$ François Eudes de Mézeray, Histoire de France depuis Faramond jusqu'à Louis le Juste, Bd. 3, Paris 1685, S. 122, Zitat S. 1345, zit. n. VIENNOT, Femmes d'État, S. 96, Anm. 45.

${ }^{8}$ Louis LACOUR, Art. „Anne d'Este et de Ferrare, duchesse de Guise«, in: Nouvelle biographie générale, Bd. 22, Paris 1859, ND 1966, Sp. 783; Carlo Novellis, Dizionario delle donne celebri piemontesi, che nacquero, vissero, morirono od ebbero relazione con questa terra le quali acquistarono in qualsiasi modo fama, Turin 1853; C. M. PILLET, Art. »Ferrare (Anne de) «, in: Biographie universelle (Michaud) ancienne et moderne, Bd. 13, Paris 1855, S. 609.

9 Jules MICHELET, Renaissance et Réforme. Histoire de France au XVI' siècle (1855-1867), Paris 1982, S. 553, 590, s. auch S. 589; Édouard FREMY, Essai sur les diplomates du temps de la Ligue. D'après des documents nouveaux et inédits, Paris 1873, ND 1971; Lucien ROMIER, Les origines politiques des guerres de religion, 2 Bde., Paris 1913-1914. 
d'Amboise, mori nel 1607 « $^{10}$. Der einige Zeit zuvor in den $\gg$ Principesse italiane nella storia d'altri paesi« erschienene Artikel von Oreste Ferdinando Tencajoli ist zwar sehr viel ausfuhrlicher und aufschlußreicher, wirklich neue Erkenntnisse bietet jedoch auch er nicht, ebensowenig wie der Anna gewidmete Eintrag im »Dictionnaire de biographie française « von 1975, und selbst in Neuerscheinungen zur Geschichte der Frauen in Frankreich fällt der Name der Prinzessin kaum ${ }^{11}$. In einer allgemeinen Darstellung schließlich eine Erwăhnung derjenigen zu suchen, die zu den einflußreichsten Damen des französischen Hofes in der zweiten Hălfte des 16. Jahrhunderts gehörte, bedeutet, "se fatiguer les yeux pour rien«, um noch einmal mit Viennot zu sprechen ${ }^{12}$.

In den letzten Jahren haben die Arbeiten von Éliane Viennot und Penny Richards dazu beigetragen, daß Anna d'Este und die anderen Frauen der Guise nicht vergessen werden. Seit 2004 hat die italienische Prinzessin einen Eintrag auf der Internetseite der Société internationale pour l'étude des femmes de l'Ancien Régime, und die Société de l'émulation de l'arrondissement de Montargis widmete ihr einen Sonderband ihres Bulletin ${ }^{13}$. Doch verdeutlichen die jüneren Veröffentlichungen auch die Problematik des Umgangs mit wenig erforschten Frauenbiographien. So führen etwa Jessica Munns und Penny Richards in einem gemeinsamen Aufsatz eine Untersuchung zu der italienischen Prinzessin durch, die zu der interessanten Erkenntnis gelangt, Anna habe "cultural gender conventions" systematisch ausgenutzt, um politischen Einfluß zu erlangen, etwa während der Prozession in der Kirche von Meulan, wo sie einen »discourse of femininity « mit einem »discourse of power« kombiniert habe, um ihr Anliegen mit größerem Nachdruck vorbringen zu können. Doch stützen sich auch diese beiden Autorinnen in ihrem Aufsatz, von einer Ausnahme abgesehen, auf die üblichen Memoiren der Zeitgenossen, teilweise auf deren Nacherzählung durch Historiker des 20. Jahrhunderts, denen sie die

${ }^{10}$ Francesco ORESTANO (Hg.), Eroine, ispiratrici e donne di eccezione, Mailand 1940, S. 168.

11 Oreste Ferdinando Tencajoll, Anna d'Este. Duchessa di Guisa e di Nemours (15481607), in: DERS., Principesse italiane nella storia d'altri paesi, Rom 1933, S. 222-233; François d'AMAT, Art. »Este, Anne d'«, in: Dictionnaire de biographie française, Bd. 13, Paris 1975, Sp. 74-76. Kurz erwăhnt wird Anna d'Este in: Scarlett BEAUVALETBouTouYRIE, Les femmes à l'époque moderne (XVI ${ }^{e}-X{ }^{2} I^{e}$ siècles), Paris 2003, S. 222f.; Dominique GodineaU, Les femmes dans la société française, $\mathrm{XVI}^{e}-\mathrm{XVIII}{ }^{e}$ siècle, Paris 2003, S. 90.

${ }^{12}$ Éliane VIENNOT, Marguerite de Valois. Histoire d'une femme, histoire d'un mythe, Paris 1993, S. 11.

${ }^{13}$ Penny RichardS, Art. "Nemours, Anne d'Este, duchesse de Guise et de" (2004), in: Dictionnaire des femmes de l'Ancienne France, unter: www.siefar.org/DictionnaireSIEFAR/ SFEste.html; Huguette LELOUP, Anne d'Este (1531-1607). Fille aînée de Renée de France, Duchesse de Guise puis duchesse de Nemours, Dame de Montargis. Sonderband des Bulletin de la Société d'émulation de l'arrondissement de Montargis, 3. Ser., 119 (2002). Hierbei handelt es sich allerdings nicht um einen wissenschaftlichen Beitrag. 
immer gleichen Episoden entnehmen und hinter denen die Person der Herzogin fast völlig verschwindet. Nicht nur die Tatsache, daß die Frage nach Glaubwürdigkeit und Intentionen der Texte nur auf ungenügende Weise gestellt wird, kőnnte an diesem Vorgehen kritisiert werden, sondern auch die Instrumentalisierung eines Frauenlebens, dem die außergewöhnlichen Ereignisse entnommen werden, das ansonsten aber farblos bleibt ${ }^{14}$.

Dem Anna d'Este gewidmeten Artikel im $»$ Dizionario biografico degli Italiani« schließlich kommt zwar das Verdienst zu, erstmals eine umfassende Darstellung zumindest der politischen Aktivitäten der Prinzessin versucht zu haben, doch ist auch dieser Artikel nicht vor den Nachteilen eines allgemeinen Lexikoneintrags gefeit. Dies kann etwa anhand der Darstellung von Annas Rolle fur die Ereignisse um die Bartholomäusnacht demonstriert werden. Unter Ignorierung der modernen Forschung folgt der Autor hier den Darstellungen der Zeitgenossen und erklärt die Herzogin von Nemours kurzerhand zum ausführenden Arm der Königinmutter und zur Auftraggeberin des Anschlags auf Coligny. Doch zumindest hat dieser Eintrag im "Dizionario « dazu geführt, daß Luciano Chiappini in der Überarbeitung seiner Geschichte über "Gli Estensi« von 1967 die Prinzessin berlicksichtigte, welcher in der neuen und erweiterten Auflage des Buches ganze zweieinhalb Seiten gewidmet $\operatorname{sind}^{15}$.

Das Schicksal, »un des personnages les plus importants de la scène politique française « ihrer Zeit gewesen zu sein, von der modernen Forschung aber weitgehend ignoriert zu werden, teilt Anna d'Este mit vielen ihrer Standesgenossinnen ${ }^{16}$. Zwar sind Fürstinnen in den Geschichtswerken des Mittelalters ebenso präsent wie Fürsten, und seit dem 16. Jahrhundert finden sich in den französischen Katalogen vorbildlicher Frauen neben antiken vermehrt auch zeitgenössische Beispiele, doch wurden die weiblichen Protagonisten spätestens seit der Dritten Republik, als die Geschichtsschreibung $>$ Wissenschaftlichkeit für sich entdeckte und die Trennung zwischen vermeintlich seriöser Historik und belletristischer Biographik vollzog, aus den Büchern verdrăngt. Bis heute sind in den Regalen der Buchhandlungen weitaus mehr Männern gewidmete Biographien zu finden ${ }^{17}$. Da sich die deutschsprachige Frauenge-

14 Jessica MUNNS, Penny RICHARDS, Exploiting and destabilizing Gender Roles: Anne d'Este, in: French History 6 (1992) S. 206-215, Zitate S. $210 \mathrm{f}$.

${ }^{15}$ Matteo SANFILIPPO, Art. »Este, Anna d'«, in: Dizionario biografico degli Italiani, Bd. 43, Rom 1993, S. 315-320; Luciano CHIAPPINI, Gli Estensi. Mille anni di storia, Ferrara 2001, S. 345-348.

${ }^{16}$ VIENNOT, Femmes d'État, S. 81. Die Stelle bezieht sich auf Anna d'Este.

${ }^{17} \mathrm{Vgl}$. die Zahlen in: Angelika SCHASER, Bedeutende Männer und wahre Frauen. Biographien in der Geschichtswissenschaft, in: Irmela von der LÜHE, Anita RUNGE (Hg.), Biographisches Erzählen, Stuttgart, Weimar 2001, S. 137-152, hier S. 141-143; Susan Mosher STUARD, Fashion's Captives: Medieval Women in French Historiography, in: DIES. (Hg.), Women in Medieval History and Historiography, Philadelphia 1987, S. 59-80, hier 
schichte des 20. Jahrhunderts zunächst für Frauen der unteren Gesellschaftsschichten interessierte und mit den Bereichen Arbeit, Religion und Frömmigkeit auseinandersetzte, wurden die Damen der Hocharistokratie anfangs auch von ihr vemachlässigt. Später konzentrierte sich die Adelsforschung vor allem auf Eheschließungen und die damit verbundenen Hochzeitsfeierlichkeiten, so $\mathrm{da} ß$ uber das Leben dieser Frauen kaum etwas bekannt ist ${ }^{18}$. Ein Blick in das Inhaltsverzeichnis einer jüngeren Veröffentlichung zur Geschlechtergeschichte der Fruhen Neuzeit zeigt, daß der Schwerpunkt der Forschung auch weiterhin auf »Religiosität und Spiritualität« sowie den »Ordnungen des Hauses und des Dorfes« liegt, während den Fürstinnen nur ein kleiner Teil des Augenmerks gilt ${ }^{19}$.

$\mathrm{Zu}$ diesen Problemen kommt für Anna d'Este erschwerend ihre Mittelstellung zwischen italienischem und französischem Interesse hinzu. Während sich nämlich im 19. Jahrhundert, als viele weibliche Angehörige der europäischen Höfe eine Lebensbeschreibung erhielten, französische Biographen, die nicht selten selbst den Titel eines Baron oder Grafen trugen, ausschließlich mit französischem Adel entstammenden Damen beschäftigten, fanden italienische und anglo-amerikanische Historikerinnen und Historiker ihr Betätigungsfeld in der Renaissance Italiens und ihren Măzeninnen ${ }^{20}$. Bis heute macht sich diese Akzentsetzung bemerkbar, wie ein Blick in zwei parallel angelegte und zeitgleich veröffentlichte Sammlungen von Biographien zeigt, die beide den Untertitel »Dichterinnen, Malerinnen, Mäzeninnen« tragen. Während die Beiträge in dem von Margarete Zimmermann und Roswitha Böhm betreuten Band über französische Frauen etwa zur Hälfte Königinnen oder Angehörigen

S. 60, 66; Margarete ZIMMERMANN, Gender, Gedächtnis und literarische Kultur. Zum Projekt einer Autorinnen-Literaturgeschichte bis 1750, in: Renate KROLL, DIES. (Hg.), Gender Studies in den romanischen Literaturen: Revisionen, Subversionen, Bd. 1, Frankfurt a. M. 1999, S. 29-55, hier S. 35; Gianna POMATA, Storia particolare e storia universale: In margine ad alcuni manuali di storia delle donne, in: Quaderni storici 25 (1990) S. 341-385, hier S. 362-364.

${ }^{18}$ Anne CONRAD, Frauen- und Geschlechtergeschichte, in: Aufriß der Historischen Wissenschaften, Bd. 7: Michael MAURER (Hg.), Neue Themen und Methoden der Geschichtswissenschaft, Stuttgart 2003, S. 230-293, hier S. 282f.; Jörg ROGGE, Nur verkaufte Töchter? Überlegungen zu Aufgaben, Quellen, Methoden und Perspektiven einer Sozial- und Kulturgeschichte hochadeliger Frauen und Fürstinnen im deutschen Reich während des späten Mittelalters und am Beginn der Neuzeit, in: Cordula NolTE u.a. (Hg.), Principes. Dynastien und Höfe im späten Mittelalter, Stuttgart 2002, S. 235-276, hier S. 237f.

${ }^{19}$ Heide WUNDER, Gisela ENGEL (Hg.), Geschlechterperspektiven. Forschungen zur Frithen Neuzeit, Königstein i. T. 1998, S. 5-8.

${ }^{20}$ Als Beispiele seien genannt: Auguste-Théodore de GIRARDOT, Procès de Renée de France, Dame de Montargis, contre Charles IX, Nantes o.J. [1858]; Gabriel de PIMODAN, La mère des Guises. Antoinette de Bourbon, 1494-1583, Paris 1925; Julia CARTWRIGHT, Isabella d'Este, Marchioness of Mantua, 1474-1539. A Study of the Renaissance, 2 Bde., London 1903; Maud F. JERROLD, Vittoria Colonna. With some Account of her Friends and her Times, London 1906. 
des höchsten Adels gewidmet sind, beschäftigt sich der von Irmgard OsolsWehden herausgegebene Band über italienische Frauen zum überwiegenden Teil mit nichtadligen Künstlerinnen ${ }^{21}$. Es erstaunt daher kaum, daß die ersten Quellensammlungen über die ebenfalls sowohl in Italien als auch in Frankreich wirkende Renée de France und ihre Töchter von einem Deutschen, Ernst von Münch, zusammengestellt wurden, der sich weder ausschließlich den Damen der italienischen Renaissance noch denen des französischen Hofes verpflichtet fühlte ${ }^{22}$.

Die von Jörg Rogge hinsichtlich von Fürstinnen des deutschsprachigen Raumes postulierten Forschungsdesiderate, die auch für die franzősischen und italienischen Standesgenossinnen Gültigkeit besitzen, konzentrieren sich auf materielle Rahmenbedingungen und die soziale Praxis der Lebensfuhrung hochadliger Frauen sowie auf Normen und kulturelle Leitbilder ${ }^{23}$. Wenn in diesen Bereichen auch große Forschungslücken bestehen, so sind doch im Zuge der Intensivierung der Patronageforschung in den letzten zwei Jahrzehnten weibliche Angehörige des Adels vermehrt in das Blickfeld der Geschichtswissenschaft gerückt ${ }^{24}$. Die Publikationen von Kristen Neuschel und Sharon Kettering zu den Klientelbeziehungen und Haushalten von adligen Frauen in Frankreich sowie Untersuchungen zu bekannteren und unbekannteren Fürstinnen wie Margarete von Navarra und Margarete von Valois oder Jeanne de Gontault und Chrétienne d'Aguerre zeichnen ein differenzierteres Bild von den Damen des 16. Jahrhunderts, die den Familienbesitz verwalteten, sich um Einfluß bei Hofe und vorteilhafte Heiraten für den Nachwuchs bemühten und aktiv am politischen Geschehen der Zeit teilnahmen ${ }^{25}$. Historikerinnen und Historiker haben in den vergangenen Jahren über die Bedeutung adliger Frauen aus ökonomischer und religiöser Sicht geforscht, der Witwenstand als »espace de liberté« ist entdeckt worden, und die Göttinger Residen-

${ }^{21}$ Margarete ZIMMERMANN, Roswitha BÖHM (Hg.), Französische Frauen der Frühen Neuzeit. Dichterinnen, Malerinnen, Măzeninnen, Darmstadt 1999; Irmgard OSOLS-WEHDEN (Hg.), Frauen der italienischen Renaissance. Dichterinnen, Malerinnen, Mäzeninnen, Darmstadt 1999.

${ }^{22}$ Ernst MƠNCH, Erinnerungen an ausgezeichnete Frauen Italiens, ihr Leben und ihre Schriften, Bde. 1-2: Renea von Este und ihre Töchter: Anna von Guise, Lukrezia von Urbino und Leonore von Este, Aachen, Leipzig 1831-1833; DERS. (Hg.), Denkwürdigkeiten zur Geschichte der Häuser Este und Lothringen im XVI. und XVII. Jahrhundert, bestehend aus ungedruckten Briefen, Memoiren, Staatsrelationen, Bd. 1, Stuttgart 1840.

${ }^{23}$ ROGGE, Töchter, S. 244.

${ }^{24} \mathrm{Vgl}$. Heiko DROSTE, Patronage in der Frühen Neuzeit - Institution und Kulturform, in: Zeitschrift furr historische Forschung 30 (2003) S. 555-590.

${ }^{25}$ Barbara STEPHENSON, The Power and Patronage of Marguerite de Navarre, Aldershot, Burlington 2004; VIENNOT, Marguerite de Valois; Robert J. KALAS, The Noble Widow's Place in the Patriarchal Household: The Life and Career of Jeanne de Gontault, in: Sixteenth Century Journal 24 (1993) S. 519-539; Claudine ALLAG, Chrétienne d'Aguerre, comtesse de Sault, Paris 1995. 
zenkommission hat eine ihrer Konferenzen dem »Frauenzimmer" und mithin dem Alltag von Frauen bei Hofe gewidmet ${ }^{26}$. Dennoch sind diese Fürstinnen in allgemeinen Darstellungen zu den Religionskriegen oder zur Geschichte Frankreichs im 16. Jahrhundert auch weiterhin nicht $\mathrm{zu}$ finden ${ }^{27}$.

Angesichts der unbefriedigenden Literaturlage waren längere Forschungsaufenthalte in verschiedenen Archiven sowohl in Frankreich als auch in Italien nötig, um die Basis für eine Beschăftigung mit der italienischen, am französischen Hofe lebenden Anna d'Este zu legen. Wichtigste Anlaufstelle war das Staatsarchiv in Modena, wo die Familienpapiere der Este zu finden sind. Nachdem Ferrara 1598 an den Kirchenstaat gefallen war, hatte sich der Erbe des Herzogs, der aus einer Nebenlinie stammende Cesare d'Este, in sein Reichslehen Modena zurïckgezogen und neben seinem Hof auch die Kunstsammlung und das Archiv mitgenommen. Seit dieser Zeit liegen in Modena alle offiziellen Papiere, Verträge und Urkunden der Familie, außerdem die Korrespondenzen der Gesandten, welche die Herzöge an den Höfen inner- und außerhalb Italiens beschäftigten, sowie der gesamte private Briefverkehr, soweit er erhalten ist ${ }^{28}$. Schwieriger stellt sich die Situation in den Pariser Archiven und Bibliotheken dar. Obwohl Anna d'Este den größten Teil ihres Lebens am französischen Hof verbrachte, ist die Suche nach sie betreffenden Dokumenten hier weitaus mulhsamer, da die Archive der Adelsfamilien entweder auf die verschiedenen Fonds der Nationalbibliothek verteilt sind oder aber während der Revolution geplündert oder zerstört wurden. So sind etwa die Papiere der Guise über den Fonds français und die Nouvelles acquisitions sowie verschiedene Sammlungen wie die von Clairambault und Dupuy verstreut. Auch die die Guise betreffenden Notariatsakten im Pariser Nationalarchiv sind lückenhaft, was vor allem für die Jahre bis 1563 gilt, als Anna den

${ }^{26}$ Sara CHAPMAN, Patronage as Family Economy: The Role of Women in the Patron-Client Network of the Phelypeaux de Pontchartrain Family, 1670-1715, in: French Historical Studies 24 (2001) S. 11-35; S. Amanda EURICH, The Economics of Power: The Private Finances of the House of Foix-Navarre-Albret during the Religious Wars, Kirksville 1994; Rosine LAMBIN, Femmes de paix. La coexistence religieuse et les dames de la noblesse en France, 1520-1630, Paris u.a. 2003; Danielle HAASE-DuBOSC, Les femmes, le droit et la jurisprudence dans la première moitié du XVII siècle, in: Kathleen WILSON-CHEVALIER, Éliane VIENNOT (Hg.), Royaume de fémynie. Pouvoirs, contraintes, espaces de liberté des femmes, de la Renaissance à la Fronde, Paris 1999, S. 51-60, Zitat S. 57; Andrea LANGER, Fürstliche und adlige Witwen in der Frühen Neuzeit. Zwischen Fremd- und Selbstbestimmung, in: Mitteilungen der Residenzen-Kommission der Akademie der Wissenschaften zu Gorttingen 11/2 (2001) S. 23-27; Jan HIRSCHBIEGEL, Werner PARAVICINI (Hg.), Das Frauenzimmer. Die Frau bei Hofe in Spätmittelalter und früher Neuzeit, Stuttgart 2000.

${ }^{27}$ Als Beispiele seien genannt: Janine GARRISSON, Guerre civile et compromis, 1559-1598, Paris 1991; Arlette JoUANNA, La France du XVI' siècle, 1483-1598, Paris ${ }^{2} 1997$.

${ }^{28}$ Filippo VALENTI, Einleitung zu: Archivio di Stato di Modena: Anchivio segreto Estense. Sezione "Casa e Stato«, Inventario, Rom 1953, S. VII-LI; DERS., Archivio di Stato di Modena, in: Piero D'ANGiolini, Claudio PAvone (Hg.), Guida generale degli Archivi di Stato italiani, Bd. 2, Rom 1983, S. 993-1080. 
Herzogintitel trug ${ }^{29}$. Weitaus besser dokumentiert ist hingegen ihre Zeit als Herzogin von Nemours. Da ihr Gemahl aus einer Nebenlinie des Hauses Savoyen stammte, befindet sich heute ein großer Teil von Annas persönlichen Papieren im Staatsarchiv Turin: Korrespondenzen und offizielle Dokumente im Fonds »Principi del Genevese e di Nemours« an der Piazza Castello, Rechnungen und Rechnungsbücher im Archvio camerale Piemonte in der Via Piave $^{30}$.

Für die Darstellung des alltäglichen Lebens der Herzogin von Guise und von Nemours sind neben den Inventaren ihrer Schlösser und Häuser vor allem Dokumente der Buchhaltung, wie Rechnungen, Quittungen, Schuldscheine und Verzeichnisse der Einnahmen und der Ausgaben von Bedeutung. Offizielle Urkunden und Verträge, die Heiraten, Erbschaften, Schenkungen, Käufe und Verkäufe dokumentieren, sowie die Akten der von Anna geführten Prozesse beschreiben hingegen die weniger alltäglichen Ereignisse. Die in ihrem Umfang größte und in ihrer Bedeutung für diese Arbeit wichtigste Quellengruppe stellen jedoch Briefe dar. Persönliche Korrespondenz war im 16. Jahrhundert noch kein Mittel der Selbstspiegelung und mithin fur die Schreiberin oder den Schreiber auch keine Möglichkeit, Gefühle zu äußern. Wie Maria Pia Fantini es für die Briefe der Cassandra Chigi dargelegt hat, sind auch in den Schreiben der Prinzessin weder das Schriftbild noch die formelhaften Äußerungen von Freude, Trauer oder Mitleid Offenbarungen ihrer swirklichen Geistesverfassung, und nur selten ist aus ihren Briefen Vergnügen oder Wut $\mathrm{zu}_{\text {ersehen }}{ }^{31}$. So ist es weniger die Art und Weise, wie ein bestimmter Sachverhalt dargestellt wird, als vielmehr, ob von ihm überhaupt die Rede ist, und wenn ja, wann. Als etwa kurz hintereinander erst Annas Bruder, dann ihre Schwester starb, verfaßte die Herzogin ihre Beileidsschreiben an deren Erben erst, nachdem sie den ihrer Meinung nach ihr selbst zustehenden Teil der französischen Hinterlassenschaft in Besitz genommen hatte, nämlich Monate

${ }^{29}$ Werner PARAVICINI, Die Nationalbibliothek in Paris. Ein Führer zu den Beständen aus dem Mittelalter und der Fruhen Neuzeit, München 1981; DERS., Das Nationalarchiv in Paris. Ein Führer zu den Beständen aus dem Mittelalter und der Frühen Neuzeit, München 1980. Vgl. Charles-Victor LANGLOIS, Les hôtels de Clisson, de Guise et de Rohan-Soubise au Marais, Paris 1922, S. 20.

${ }^{30}$ Isabella MASSABÒ RICCI, Archivio di Stato di Torino, in: Paola CARUCCI u.a. (Hg.), Guida generale degli Archivi di Stato italiani, Bd. 4, Rom 1994, S. 361-641; DIES., Maria GATTULlo (Hg.), L'Archivio di Stato di Torino, Fiesole 1994.

${ }^{31}$ Maria Pia FANTINI, Lettere alla madre di Cassandra Chigi (1535-1556): grafia, espressione, messaggio, in: Gabriella ZARRI (Hg.), Per lettera. La scrittura epistolare femminile tra archivio e tipografia, secoli XV-XVII, Rom 1999, S. 111-150. Auch die Briefe der Dames des Roches bieten keine Einblicke in das Gefuhlsleben der Verfasserinnen: ZIMMERMANN, Salon, S. 173. Vgl. Ingrid KASTEN u.a. (Hg.), Kulturen der Gefühle in Mittelalter und Früher Neuzeit, Stuttgart, Weimar 2002. 
später $^{32}$. Der Bedeutung dieser Briefe soll hier in einem eigenen, mit "Taktik und Rhetorik « uberschriebenen Absatz Rechnung getragen werden. Anhand eines ihrer Schreiben an den Bruder Alfonso werden die argumentativen und sprachlichen Strategien untersucht, derer sich die Prinzessin bediente, um den Korrespondenzpartner von ihren Ansichten zu überzeugen und das von ihr Gewünschte zu erlangen.

Neben Archivmaterial wurden für die vorliegende Arbeit auch publizierte Quellen herangezogen, wie beispielsweise die Briefe der Katharina von Medici oder die des Kardinals von Lothringen, Annas Schwager. Die wichtigste, aber auch sehr problematische Gruppe der veröffentlichten Quellentexte stellen die Gesandtenberichte dar. Die Korrespondenzen der Botschafter aus England und Spanien, Savoyen und Florenz liegen ganz oder teilweise vor, ebenso die Briefe des Nuntius sowie die Relazioni der venezianischen Gesandten. Da diese Männer sich meist in einer prekären Situation befanden, abhängig waren von ständig wechselnden politischen Allianzen und von der Gunst, die ihre Brotgeber bei Hofe genossen, neben der Erfüllung ihrer Pflichten auch auf die Vertretung ihrer eigenen Interessen bedacht und in ihrer Berichterstattung nur selten objektiv, muß diese Quellengruppe mit besonderer Vorsicht behandelt werden ${ }^{33}$. Daneben berichten Memoiren und zeitgenössische Geschichtsbücher sowie literarische Werke und bildliche Darstellungen über die Herzogin von Guise und Nemours, wobei von Lobgesängen bis zu offensichtlichen Verleumdungen alle Sichtweisen vertreten sind. Da Selbstaussagen der Prinzessin größtenteils fehlen, ist man bei der Interpretation auf diese stark divergierenden Wahrnehmungen der Zeitgenossen angewiesen.

\section{Zur Problematik der Biographie}

"Wer Biograph wird, verpflichtet sich zur Lüge, zur Verheimlichung, Heuchelei, Schönfärberei und selbst zur Verhehlung seines Unverständnisses, denn die biographische Wahrheit ist nicht zu haben, und wenn man sie hătte, wäre sie nicht zu brauchen«, schreibt Sigmund Freud im Jahre $1936^{34}$. Obwohl ein großer Teil der heute erscheinenden Lebensbeschreibungen auch weiterhin

${ }^{32}$ Anna d'Este an Cesare d'Este und Virginia de' Medici, 22. Apr. 1598, Paris, in: ASM, Canc. duc., Cart. prin. est. 1458/22, 1459/23. Alfonso war im Oktober 1597, Lucrezia im Februar 1598 gestorben.

${ }^{33}$ Eine Einschätzung der ausländischen Gesandten am französischen Hof im 16. Jh.: Lucien ROMIER, Le royaume de Catherine de Médicis. La France à la veille des guerres de religion, Bd. 1, Paris 1925, ND 1978, S. XXIII-XXVI.

${ }^{34}$ Sigmund Freud an Arnold Zweig, 31. Mai 1936, Wien, in: Sigmund FrEUD, Briefe 18731939, hg. v. Ernst FrEUD, Lucie FrEUD, Frankfurt a. M. ${ }^{2} 1960$, S. 445. 
nach der swahren Person und Persönlichkeit ihrer Protagonisten sucht, beginnt sich in der Geschichtswissenschaft die Einsicht durchzusetzen, daß die historische Biographik ihren absoluten Wahrheitsanspruch und ihre Suche nach Fakten, Stringenz und Kohärenz aufgeben sollte. So hat etwa Hans Erich Bödeker festgestellt, daß es theoretisch zahlreiche unterschiedliche Biographien zu einer einzigen historischen Figur geben musse, womit er auf die oftmals widerspruchlichen Darstellungsarten verweist, die zu ein und derselben Person denkbar wären ${ }^{35}$. Tatsächlich besteht eine der schwierigsten Aufgaben bei der Beschreibung eines Lebensweges darin, die vielen unterschiedlichen Bilder der zu schildernden Person, seien sie geschaffen von beobachtenden Zeitgenossen oder von berichtenden Geschichtsschreibem oder aber verbreitet von der Person selbst, in einem Text zu vereinen, ohne der Versuchung $\mathrm{zu}$ erliegen, eventuell divergierende Tatbestände abzustimmen und den Eindruck der Kohärenz zu erwecken. Verschiedene Biographien zu ein und demselben Menschen könnten daher viel zur Erkenntnis über ihn und seine Zeit beitragen, denn jede würde anderen Quellen folgen, einen anderen Blickwinkel einnehmen und zu anderen Ergebnissen gelangen als die anderen.

Die Bücher und Aufsätze über Katharina von Medici bieten hierfür ein gutes Beispiel. So beschreibt etwa die Darstellung von Jean-Hippolyte Mariéjol, die erste umfassende uberhaupt, die Ereignisse ihres Lebens in chronologischer Ordnung, versucht sich aber auch an einer psychologischen Deutung. In seiner Einleitung weist der Autor darauf hin, Katharinas Biographie zu schreiben, 》et non l'histoire de son temps«. Eben dies tut Robert Knecht in seinem in der Reihe mit dem sprechenden Namen "Profiles in Power« erschienenen Buch. Es ist eigentlich keine Biographie, sondern ein Handbuch zur Geschichte der Religionskriege, behandelt es doch fast ausschließlich die politische Situation der Zeit und kommt nur sehr peripher auf seine Hauptperson zu sprechen. Einen weiteren Ansatz bietet ein Aufsatz von Sheila Ffolliott, der die Darstellung und Selbstdarstellung der Königinmutter untersucht und somit ein wieder anderes Licht auf das Leben der "sschwarzen Witwe» wirft. Die aktuellste Untersuchung schließlich bringt schon in ihrem Untertitel die angesprochene Problematik zum Ausdruck, denn Janine Garrisson nennt ihre Biographie der Katharina von Medici "L'impossible harmonie«. Die erwähnten Arbeiten nehmen unterschiedliche Positionen ein und kommen zu unterschiedlichen Ergebnissen, tragen aber alle einen Teil zum Verständnis dieser umstrittenen Persönlichkeit und ihrer nicht einfach zu erklärenden Zeit bei. Vor allem aber verdeutlichen diese verschiedenen Ansätze, daß jede Biographie Konstruktion ist, und daß daher der Versuch, eine vermeintlich objektive Interpretation des

${ }^{35}$ Hans Erich BODEKER, Biographie. Annäherungen an den gegenwărtigen Forschungs- und Diskussionsstand, in: DERS. (Hg.), Biographie schreiben, Göttingen 2003, S. 9-63, hier S. 51. Vgl. Jacques REVEL, La biographie comme problème historiographique, in: BÓDEKER (Hg.), Biographie schreiben, S. 327-348, hier S. 345-347. 
Lebensweges eines Menschen zu liefern, von vornherein zum Scheitern verurteilt sein $\mathrm{muB}^{36}$.

Die erste wissenschaftliche Biographie der Anna d'Este sieht sich Schwierigkeiten gegenuber, die bei der Darstellung eines weitgehend erforschten Lebensweges, wie dem der Katharina von Medici, nicht mehr in dem Maße auftauchen. Deshalb ist dies ein janusköpfiges Buch. Zum einen hat es den Anspruch, Ereignisse aus dem Leben seiner Protagonistin ausfuhrlich darzustellen, um Historikern und Historikerinnen, die uber andere Themenkomplexe arbeiten, eine verläßliche Auskunft über diese Fürstin zu bieten, deren Name immerhin in den Personenverzeichnissen fast aller Bücher uber die Zeit der französischen Religionskriege auftauch ${ }^{37}$. Zum anderen soll es sich der Rolle adliger Frauen für die Verwaltung von Familienbesitz und die Organisation der maison oder ihrer Bedeutung für Erziehung und Karriereplanungen für den Nachwuchs im Falle einer frühen Witwenschaft widmen. Die Frage, ob die aus der Untersuchung von Annas Leben resultierenden Erkenntnisse auch auf ihre Standesgenossinnen ubbertragen werden können, ist nicht einfach zu beantworten. Als Tochter einer fille de France und mithin Cousine ersten Grades Heinrichs II., als Italienerin in Frankreich, als Gemahlin eines radikalen Katholiken, aber Tochter einer bekennenden Hugenottin, weist ihr 'Fall Besonderheiten auf, die sich in den Lebenswegen anderer Fürstinnen nicht finden.

Ähnliche Überlegungen hinsichtlich der Vergleichbarkeit von Handlungsspielräumen ihrer Protagonistin stellt auch die Biographin der Margarete von Navarra an. Als Schwester des Königs und weiblicher pair de France habe Margarete uber außergewöhnliche Möglichkeiten verfugt und daher in Frankreich eine sanomalous position « eingenommen; dennoch könne eine Beschäftigung mit ihrem Leben auch Erkenntnisse über ihre Standesgenossinnen forr$\operatorname{der}^{38}$. Das Beispiel zeigt, daß trotz einiger Übereinstimmungen von allgemeinen Aussagen über das Leben dieser Frauen Abstand genommen werden sollte, und so versteht sich das vorliegende Buch auch nicht als repräsentative Studie über adliges Frauenleben am französischen Hof der Zeit, wohl aber als eines der Puzzleteile, aus denen sich unser Bild von diesem Hof zusammensetzt. Es folgt somit der von Jacques Le Goff geäußerten Forderung, »die allgemeine historische Bedeutung eines individuellen Lebens aufzuzei-

\footnotetext{
${ }^{36}$ Jean-Hippolyte MARIÉJOL, Catherine de Médicis, 1519-1589 (1920), Paris 1979, Zitat S. 9; Robert J. KNECHT, Catherine de' Medici, London, New York 1998; Sheila FFolLIOTT, Catherine de' Medici as Artemisia: Figuring the Powerful Widow, in: Margaret W. FERGU. SON u.a. (Hg.), Rewriting the Renaissance. The Discourses of Sexual Difference in Early Modern Europe, Chicago, London 1986, S. 227-241; Janine GARRISSON, Catherine de Médicis. L'impossible harmonie, Paris 2002.

${ }^{37}$ Als Beispiele seien genannt: Jean-Pierre BABELON, Henri IV, Paris 1982; Ivan CloulAS, Philippe II, Paris 1992.

${ }^{38}$ STEPHENSON, Power, S. 86f., 110, Zitat S. 2.
} 
gen $\aleph^{39}$. Und das heißt, anhand der Person der Herzogin einen der vielen Wege zu beschreiben, die adeliges Frauenleben im Frankreich der Religionskriege gehen konnte. Wie der als Motto zu Beginn zitierte Satz von Thomas Mann es ausdrückt: Auch wenn Anna nicht als Beispiel für die Fürstin schlechthin gelten kann, spiegelt ihre Biographie doch die Situation auch ihrer Zeitgenossinnen wider und läßt Rückschlüsse auch auf deren Lebenswege zu.

Ein Blick in neuere Untersuchungen zum französischen Adel des. 16. Jahrhunderts bestätigt diesen Ansatz. Die Arbeiten von Stuart Carroll etwa unterstreichen die Bedeutung weiblicher Mitglieder der Hocharistokratie innerhalb des Netzwerkes ihrer Familien sowie die enge Zusammenarbeit von Fürst und Fürstin, wenn es um die Vertretung ihrer Interessen vor dem König oder, wie im Falle des Herzogs von Guise, den Mitgliedern der Pariser Ligue ging ${ }^{40}$. Die Aufgabenbereiche der Jeanne de Gontault ähneln denen der Anna d'Este, auch sie kümmerte sich nach dem Tod ihres Gemahls um die Finanzen des Sohnes, tătigte Landverkäufe und setzte sich mit Pächtern auseinander, arrangierte Heiraten fur ihre Kinder und fungierte als deren Beraterin, sie trat als Klägerin vor Gericht auf und pflegte als Hofdame die Beziehungen zur Köni$\operatorname{gin}^{41}$. Selbst über Epochen- und Ländergrenzen hinweg lassen sich Übereinstimmungen hinsichtlich des Lebensweges dieser Frauen feststellen, wie die Forschungen von Christiane Klapisch-Zuber über Florentinerinnen der Frühen Neuzeit, das Beispiel der Anna von Dänemark oder Untersuchungen zu den Damen der Familie Phélypeaux de Pontchartrain im Frankreich Ludwigs XIV. zeigen $^{42}$. So kann aus der Biographie der Anna d'Este viel über adliges Frauenleben gelernt werden, auch wenn sie als in Frankreich lebende Italienerin, Enkelin eines Königs, Tochter einer der Reformation anhängenden Frau, Gemahlin und Mutter von unversöhnlichen Katholiken nicht als typische Vertreterin für die Fürstin des 16. Jahrhunderts angesehen werden sollte.

Die wissenschaftliche Biographie wirft darüber hinaus einige allgemeine Probleme auf, mit denen sich auch dieses Buch konfrontiert sieht, vor allem hinsichtlich der vermeintlichen Kontinuität und Kohärenz eines Lebens. In

${ }^{39}$ Jacques LE GOFF, Wie schreibt man eine Biographie?, in: Der Historiker als Menschenfresser. Über den Beruf des Geschichtsschreibers, Berlin 1990, S. 103-1 12, Zitat S. 105.

${ }^{40}$ Stuart CARROLL, Noble Power during the French Wars of Religion. The Guise Affinity and the Catholic Cause in Normandy, Cambridge 1998, S. 246; DERS., The Revolt of Paris, 1588: Aristocratic Insurgency and the Mobilization of Popular Support, in: French Historical Studies 23 (2000) S. 301-337, hier S. 337.

${ }^{41}$ KalAS, Widow's Place.

${ }^{42}$ Christiane KLAPISCH-ZUBER, La femme et le lignage florentin (XIV ${ }^{e}-\mathrm{XVI}^{e}$ siècles), in: Richard C. TREXLER (Hg.), Persons in Groups. Social Behavior as Identity Formation in Medieval and Renaissance Europe, Binghamton 1985, S. 141-153; Katrin KELLER, Kurfürstin Anna von Sachsen (1532-1585). Von Moglichkeiten und Grenzen einer »Landesmutter«, in: Jan HIRSCHBIEGEL, Werner PARAVICINI (Hg.), Das Frauenzimmer. Die Frau bei Hofe in Spätmittelalter und früher Neuzeit, Stuttgart 2000, S. 263-285; CHAPMAN, Patronage. 
Bezug auf Lückenlosigkeit und Stetigkeit ihres Lebensweges wird die Beschäftigung mit Anna d'Este durch zwei gegensătzliche Hindernisse erschwert: das zeitweilige Fehlen von oder aber ein Überfluß an Quellen. Für Annas Biographie eine Kontinuität zu beanspruchen, käme daher einer Verdrehung der Tatsachen gleich, denn viele Monate ihres Lebens sind nicht dokumentiert, manchmal verschwindet sie für ganze Jahre in einem sschwarzen Loch,, und es kann nicht bestimmt werden, ob die Dokumente aus dieser Zeit vernichtet wurden, verloren sind oder einfach unentdeckt in den Tiefen der Archive schlummern. Auf der anderen Seite sind manche Ereignisse durch eine derartige Fülle von Urkunden, Briefen und anderen Berichten dokumentiert, daß absichtlich Lücken gelassen und die vermeintliche Kontinuität des Lebensweges unterbrochen werden mußte. So etwa hinsichtlich der Suche nach einer Heiratsverbindung furr Anna und der in diesem Rahmen gefuhrten Verhandlungen und unendlichen Gespräche. Schon in seiner jetzigen Form beansprucht dieser Themenkomplex ein ganzes Kapitel, was die Geduld der Leserinnen und Leser zwar strapaziert, aber die Bedeutung unterstreicht, welche diese Unterredungen für die Zukunft der Prinzessin hatten, und die alternativen Möglichkeiten auslotet, die sich ihr in anderen Konstellationen geboten hätten.

Hinsichtlich der Kohärenz von Annas Persönlichkeit ist eine Aussage nur schwer zu treffen, und tatsächlich liegt der Zweck dieser Arbeit nicht darin, ein schlüssiges Bild der Prinzessin zu zeichnen, sondern den Wahrnehmungen und Darstellungen ihrer Person durch andere und, soweit die Quellen es erlauben, durch sie selbst nachzugehen. So sahen die einen in ihr als Tochter derjenigen, die ihre Residenz entgegen aller Widerstände als Zufluchtsort verfolgter Hugenotten behauptete, wenn nicht eine Anhängerin, so doch zumindest keine Gegnerin des reformierten Glaubens. Die anderen hielten die Gemahlin desjenigen, der die sogenannte Verschwörung von Amboise mit unzähligen Todesurteilen beendete und in Vassy ein Blutbad unter den Besuchern eines reformierten Gottesdienstes anrichten ließ, ebenfalls für eine unversöhnliche Katholikin. Anna hat sich hierzu nicht geäußert, und warum sollte man überhaupt einen Zusammenhang ihrer Ideen, die Schlüssigkeit ihrer Ansichten, die Übereinstimmung ihres Denkens und Handelns annehmen? Warum sollte sie nicht sowohl eine glühende Katholikin gewesen sein als auch, gemeinsam mit der Mutter, im Schloß von Montargis die Predigt gehört haben? Schließlich haben neuere Forschungen gezeigt, daß Religion im 16. Jahrhundert nicht als Gewissenssache zu betrachten sei, sondern als soziale und kulturelle Kategorie. C'est une si petite chose, antwortete etwa eine zu ihrem religiösen Glauben befragte Dame, die während der Herrschaft der Ligue in Dijon den Kontakt $\mathrm{zu}$ ihrem hugenottischen Schwager aufrecht erhalten hatte, und sowohl katholische als auch protestantische Augenzeugen der Massaker in Paris im August 1572 berichten, radikale Katholiken wie der Herzog von Guise hätten 
einigen der von ihnen so gehaßten Hugenotten das Leben gerettet ${ }^{43}$. Die Untersuchung von Netzwerken und Beziehungssystemen, in denen sich die Protagonistin einer Biographie bewegte, scheint folglich sinnvoller als der Versuch, aus lückenhaft uberlieferten Quellen eine vermeintlich schlüssige Persönlichkeit zu rekonstruieren.

Das Problem der Kohärenz stellt sich noch in anderen Zusámmenhängen, dem von Annas Zugehörigkeitsgefuhl etwa. War die Prinzessin eine Este oder eine Guise? Fuhlte sie sich der Familie ihrer Ehegatten verbunden oder der ihres Vaters? Identifizierte sie sich mit ihrem Titel oder mit dem Patronym? Pierre Bourdieu hat die Taufe und die darauffolgende lebenslange Bezeichnung einer Person mit ihrem Namen als Institution »der Totalisierung und Vereinheitlichung des Ich « bezeichnet, da die Benennung eines Individuums mit dem immer gleichen Namen eine Konstanz suggeriere, die aufgrund stăndig wechselnder Zustände und unterschiedlicher »Felder«, in denen es sich bewege, nicht existiere ${ }^{44}$. Tatsăchlich ist an den während der ersten zwanzig Jahre ihres Lebens öfter veränderten Unterschriften zu erkennen, daß die Frage ihrer Zugehörigkeit von der Prinzessin nicht von vornherein eindeutig beantwortet werden konnte. Aus den italienischen Formen »Anna da Est« und "Anna d'Este« wurde zu Beginn ihres Aufenthaltes in Frankreich, als die Identifikation mit dem Gatten und dessen Titel besonders groß gewesen zu sein scheint, "La Duchessa d'Omale«, was eine wahre Seltenheit unter den Namenszügen franzősischer Fürstinnen darstellt. Denn natürlich unterzeichnete Diane de Poitiers ihre Briefe mit »Dianne de Poytiers« und nicht mit "Die Herzogin von Valentinois«, Henriette und Catherine de Clèves schrieben "Henriette de Cleves" und "Katerine de Cleves" und nicht "Die Herzogin von Nevers« oder »Guise«, Catherine de Lorraine unterzeichnete mit ihrem Namen und nicht mit dem Titel der Herzogin von Montpensier, ebenso wie ihre Großmutter Antoinette de Bourbon. Tatsächlich war Anna nur kurze Zeit Herzogin von Aumale, und nachdem ihr Gatte das Herzogtum Guise geerbt hatte, unterzeichnete auch sie stets mit der französischen Form ihres Namens, "Anne d'Est $\ll$.

Weitere Schwierigkeiten bei der Darstellung eines Frauenlebens in Form einer Biographie entstehen hinsichtlich der Auseinandersetzung mit einem

${ }^{43}$ Mack P. HoLT, Putting Religion Back into the Wars of Religion, in: French Historical Studies 18 (1993) S. 524-551; Verhör der Marguerite Millière vor dem Stadtrat von Dijon, 2. Apr. 1590, in: S. Annette FNNEY-CROSWHITE, Engendering the Wars of Religion: Female Agency during the Catholic League in Dijon, in: French Historical Studies 20 (1997) S. 127154, Zitat S. 148; Barbara B. DIEFENDORF, Beneath the Cross. Catholics and Huguenots in Sixteenth-Century Paris, New York, Oxford 1991, S. 104; Janine GARRISSON, 1572, La Saint-Barthélemy, Brüssel 2000, S. 193. Vgl. auch Lucien FEBVRE, Margarete von Navarra. Eine Königin der Renaissance zwischen Macht, Liebe und Religion (1944), hg. v. Peter SCHÖTTLER, Frankfurt a. M. u.a. 1998.

${ }^{44}$ Pierre BOURDIEU, Die biographische Illusion (1986), in: Bios (1990) S. 75-81, Zitat S. 77. 
Konzept, das sich am besten mit dem englischen Begriff »agency « umschreiben läßt. Um es gleich vorwegzunehmen: Diese Arbeit glaubt an die Möglichkeit der Protagonistin, ihre alltägliche Existenz zu gestalten; tatsächlich geht es hier um die Darstellung bewußt genutzter Handlungsstrategien, um die Frage nach Loyalităten und die Untersuchung eines Beziehungssystems. Dennoch sollen auch die diskursiven Praktiken, derer Anna sich bediente, und der Kontext ihres Handelns dargestellt werden, um nicht den Eindruck zu vermitteln, es mit einem autonom agierenden, sich frei von Konventionen bewegenden Individuum zu tun zu haben. Beispielsweise anläßlich der Sitzung des königlichen Rates, in deren Verlauf Karl IX. eine endgültige Entscheidung hinsichtlich Schuld oder Unschuld des Admirals Coligny für den Mord am Herzog von Guise zu treffen gedachte. Während Natalie Zemon Davis beschreibt, wie aufsässige Frauen der unteren Gesellschaftsschichten männliche Verhaltensweisen imitierten und die Geschlechterverhältnisse aus Protestgründen umkehrten, unterstrich der das Anliegen seiner Schwăgerin vertretende Kardinal von Lothringen in seiner Rede vor dem König gerade Annas Hilfsbedürttigkeit als Witwe und schutzlose Frau ${ }^{45}$. Dieses Weiblichkeitsdiskurses bediente sich die Herzogin auch an anderer Stelle, etwa als sie mit dem Hinweis auf ihre eigene Schwangerschaft von ihrem Gemahl die Schonung der bei dem Massaker von Vassy anwesenden schwangeren Frauen erreichte.

In den folgenden Kapiteln sollen hauptsăchlich diejenigen Bereiche in die Untersuchung einbezogen werden, welche für die Protagonistin dieses Buches, für ihr Leben und Selbstverständnis von Bedeutung waren. Dazu gehört natürlich Annas Betătigung auf dem Gebiet der Politik in engerem Sinne, verstanden als Einflußnahme auf die Gestaltung des Hofes, die Vergabe von Ämtern und so weiter, wobei die engen Bahnen des herkömmlichen Politikbegriffes überschritten werden, etwa wenn es um Repräsentation und Rangordnung geht. Hierzu zăhlt ebenso die Sorge der Prinzessin um ihr Gefolge und die Organisation ihrer maison sowie die Tätigkeit als Verwalterin des enormen Familienbesitzes, kurz: all das, was gemeinhin unter den Begriff "Alltag" fallen würde. Diesbezüglich orientiert sich die Fragestellung in erster Linie an den grundsätzlichen, den Alltag bei Hofe betreffenden Überlegungen von Werner Paravicini ${ }^{46}$. Des weiteren soll die Fürstin in dem Beziehungsgeflecht dargestellt werden, in welchem sie sich bewegte, und das zu einem großen Teil ein rein weibliches war. Der Forderung von Gisela Bock, nicht nur die

${ }^{45}$ Natalie Zemon DAVIS, Die aufsüssige Frau, in: DiEs., Humanismus, Narrenherrschaft und die Riten der Gewalt. Gesellschaft und Kultur im fruhneuzeitlichen Frankreich (1979), Frankfurt a. M. 1987, S. 136-170.

${ }^{46}$ Werner PARAVICINI, Alltag bei Hofe, in: DeRS. (Hg.), Alltag bei Hofe. 3. Symposium der Residenzen-Kommission der Akademie der Wissenschaften in Göttingen, Sigmaringen 1995, S. 9-30. Zum Politikbegriff in Bezug auf den Hof der Frihen Neuzeit: Jeroen DuINDAM, Norbert Elias und der frühneuzeitliche Hof. Versuch einer Kritik und Weiterfuhrung, in: Historische Anthropologie 6 (1998) S. 370-387, hier S. 384. 
Beziehungen zwischen den Geschlechtern zu erforschen, sondern auch die innerhalb der Geschlechter, kommt diese Arbeit schon deshalb entgegen, weil die intensivsten und folgenreichsten Kontakte der Prinzessin die zu anderen Frauen waren, zur Mutter, zu der Studiengefährtin aus der Kindheit, zu Katharina von Medici und den Damen des Hofes ${ }^{47}$. »Freundschaft« wird dabei nach Ferdinand Tönnies als "Gemeinschaft des Geistes« und mithin als eine durch gegenseitige Vertrautheit und Achtung bestimmte soziale Beziehung zwischen zwei Menschen verstanden. Der Begriff ist somit nicht mit dem von den Zeitgenossen benutzten Wort "amitié« zu verwechseln, welches neben vielen anderen Verwendungen vor allem das Verhältnis zwischen Patron und Klient bezeichnete $^{48}$.

\section{Vorüberlegungen zur Textgestalt}

Der Aufbau der Arbeit folgt zwei verschiedenen Prinzipien. Die Kapitel sind an einer groben Chronologie der Ereignisse ausgerichtet, die Unterkapitel und einzelnen Abschnitte orientieren sich größtenteils an inhaltlichen Gesichtspunkten. So ist etwa das mit »Herzogin von Guise« betitelte Kapitel in die Gesamtheit des Textes nach chronologischen Gesichtspunkten eingebettet, die Verteilung der einzelnen Punkte auf die hier behandelten Themenkomplexe richtet sich aber ausschließlich nach ihrem Inhalt. Dies bedeutet jedoch nicht, $\mathrm{da} ß$ nicht auch an späterer Stelle auf diese Themen zurückgekommen werden kann. Viele Dinge in Annas Leben wiederholten sich - sie heiratete zweimal, zog Kinder aus zwei verschiedenen Ehen groß, fuhrte mehrere unterschiedliche Prozesse -, die Voraussetzungen stellten sich aber jeweils als so gegensätzlich dar, daß eine Zusammenfassung in vielen Fällen nicht sinnvoll erschien. Als die Prinzessin in erster Ehe eingebunden war in einen riesigen Familienclan, und Tanten, Onkel und die Großmutter mehr Verantwortung für die Kleinen übernahmen als die Eltern, war ihr Einfluß auf die Erziehung ihrer Sprößlinge, auf die Entscheidung über Heiratsverbindungen und anderes sehr viel geringer als während ihrer zweiten Ehe, als sie und ihr Gemahl, später sie ganz allein, für diese Dinge zuständig waren. Schließlich zählen bei einem so weitgefaßten Politikbegriff, wie ihn der französische Hof der Frühen Neuzeit erfordert, zu den politischen Betätigungen der Herzogin nicht nur die in dem

${ }^{47}$ Gisela BOCK, Geschichte, Frauengeschichte, Geschlechtergeschichte, in: Geschichte und Gesellschaft 14 (1988) S. 364-391, hier S. 379.

${ }^{48}$ Ferdinand TOONNIES, Gemeinschaft und Gesellschaft. Grundbegriffe der reinen Soziologie (1887), Darmstadt ${ }^{12}$ 2005; Sharon KETTERING, Friendship and Clientage in Early Modern France, in: French History 6 (1992) S. 139-158. 
mit "Die Politikerin « betitelten Kapitel behandelten Fragen, sondern auch Annas Prozesse, die Bemühungen um die Karrieren der Söhne, ihr Verhältnis zur katholischen Ligue, die Versöhnung mit Heinrich IV. und vieles mehr, so $\mathrm{daB}$ die inhaltliche Gliederung der Unterkapitel als eine sehr offene angesehen werden sollte.

Hinsichtlich der Erzählstruktur dieses Buches ist mit zwei verschiedenen Techniken gearbeitet worden. Dort, wo es um reine Analyse geht, ist die Beschreibung weniger detailliert und kommt weitgehend ohne direkte Zitate aus den Quellen aus, etwa in den Absätzen über die Erziehung der Prinzessin oder ihren Haushalt. Dieser letzte Fragenkomplex wird zunächst für die Zeit behandelt, als Anna Herzogin von Guise war, an anderer Stelle dann noch einmal für die letzte Phase ihres Lebens, als sie kaum noch ihr Pariser Hôtel de Nemours verließ, da sich die Zusammensetzung des Gefolges in jenen Jahren grundlegend änderte. In beiden Fällen werden hauptsächlich die Lohnlisten, aber auch die in Annas Rechnungsbüchern vermerkten Schenkungen zugunsten von Angehörigen der maison herangezogen. Die Präsentation der Ergebnisse verzichtet weitgehend auf illustrierende Details und widmet sich eher der Darstellung großer Linien. Der größere Teil dieses Buches verfolgt jedoch den Zweck, die Einblicke in das Leben dieser Fürstin so unmittelbar wie möglich zu gestalten. Gemäß der durch die Texte Clifford Geertz' angeregten Überzeugung, daß die Untersuchung mit ihrer Darstellungsweise meist unauflöslich verbunden sei, eine dichte Beschreibung daher einer verständlichen Beschreibung gleichkomme, werden bewußt viele Sachverhalte so detailliert wie möglich dargestellt ${ }^{49}$.

Die Vorteile dieser Technik werden etwa an dem Kapitel über die Reise der Braut nach Frankreich deutlich. Auf den ersten Blick könnte die Tatsache, daß der Abschied aus Ferrara, die Fahrt über die Alpen und die Ankunft bei Hofe zwei Drittel eines Kapitels einnehmen, Erstaunen hervorrufen. Die These lautet jedoch - und der Titel dieses Kapitels bringt es zum Ausdruck -, daß sich in der Prinzessin im Laufe dieser Reise eine Wandlung vollzogen habe, die sie zu einem Mitglied des französischen Hofes und der Familie der Guise hatte werden lassen, noch bevor sie Paris erreichte. Wie jedoch kann eine derartig subtile Veränderung anders vermittelt werden als durch die Abstimmung des Inhalts mit dem Erzăhlrhythmus? Und mithin durch eine detaillierte Beschreibung der für Anna ausgerichteten Empfänge in den Orten, durch die sie reiste, der Trauer über den Abschied von ihrem italienischen Gefolge in Grenoble, der Briefe, die sie unterwegs mit ihrem zukünftigen, noch unbekannten Gemahl tauschte, der Berichte ihrer französischen Begleiter, schließlich der erstaunten Bemerkung des ferraresischen Gesandten, welcher die Tochter seines Auftraggebers von klein auf kannte, nicht mehr die Prinzessin

${ }^{49}$ Clifford GEERTZ, Thick Description: Toward an Interpretive Theory of Culture, in: DERS., The Interpretation of Cultures. Selected Essays, New York 1973, S. 3-30. 
von Ferrara in ihr zu finden, sondern Madame d'Aumale? Und dennoch kommen nicht alle Quellen zur Sprache, mußte selbst hier eine Auswahl vorgenommen werden.

Dieses erzahlerische Verfahren ist nicht zuletzt als Versuch anzusehen, den angeblich unvermeidlichen, in der Mehrzahl der wissenschaftlichen Veröffentlichungen tatsächlich bestehenden Gegensatz zwischen narrativer und argumentativer Geschichtsschreibung aufzuweichen. Folglich verdankt dieses Buch viel den um die Beziehung von Erzählstruktur zu den durch sie transportierten Inhalten kreisenden Überlegungen von Hayden White, zwar nicht in allen Einzelheiten, vor allem nicht in seiner Theorie der Archetypen, aber doch in ihren grundsätzlichen Konsequenzen für historisches Schreiben ${ }^{50}$. Die Erkenntnis nämlich, daß die sprachliche Form auch die Ergebnisse präge, kann an keinem Beispiel so gut nachvollzogen werden wie an der eben zitierten Reise von Ferrara nach Frankreich. Denn einer gestrafften Darstellung, die kürzer bei den verschiedenen prägenden Erlebnissen verweilt wäre und sich weniger intensiv mit den Quellentexten beschäftigt hätte, wăre es unmoglich gewesen, die Verwandlung der Prinzessin zu erkennen und darzustellen.

Müßte schließlich ein Vorbild benannt werden, an dem sich diese Biographie orientierte, so würde sicher an erster Stelle das Buch uber Caesar von Christian Meier stehen, das sich als "wissenschaftliche Biographie in erzählerischer Absicht " versteht ${ }^{51}$. Mit einem grundlegenden Unterschied: Diese Arbeit über Anna d'Este widmet sich nicht in dem Maße der Darstellung der Zeitumstände, wie es in der Caesar-Biographie geschieht, obwohl sie grundsätzlich der Feststellung Goethes, die Hauptaufgabe der Biographie sei es, »den Menschen in seinen Zeitverhältnissen darzustellen«, zu folgen sucht ${ }^{52}$. Eine Behandlung der französischen Religionskriege im 16. Jahrhundert, die ja eigentlich zum Verständnis der speziellen Situation oder Person beitragen sollte, würde nämlich eine eigene Untersuchung für sich verlangen.

Da die Quellen dieser Arbeit zur einen Hälfte in italienischer, zur anderen in französischer Sprache verfaßt sind, hat sich die Notwendigkeit der Übersetzung von Titeln und Hofämtern ergeben, um zu verhindern, daß die Prinzessin einmal als "principessa«, dann wieder als »princesse « bezeichnet wird. Herzogs- und Grafentitel werden daher ubersetzt, nicht jedoch die "marchesa" und »marquise«, da das deutsche Wort »Markgräfin« die Assoziation mit dem

${ }^{50}$ Hayden WHITE, Einleitung zu: DERS., Metahistory. The Historical Imagination in Nineteenth-Century Europe, Baltimore, London 1973, S. 1-42; DERS., Der historische Text als literarisches Kunstwerk (1974), in: Christoph CONRAD, Martina KESSEL (Hg.), Geschichte schreiben in der Postmoderne. Beiträge zur aktuellen Diskussion, Stuttgart 1994, S. 123157; DERS., Die Bedeutung der Form. Erzählstrukturen in der Geschichtsschreibung (1987), Frankfurt a. M. 1990.

${ }^{51}$ Christian MEIER, Cassar, München ${ }^{2} 1986$, Zitat S. 579.

52 Johann Wolfgang GOETHE, Aus meinem Leben. Dichtung und Wahrheit, hg. v. Peter SPRENGEL, München 1985, Zitat S. 11. 
mittelalterlichen Beherrscher eines Grenzgebietes hervorrufen würde. Ebenso wurde bei den Hofämtern verfahren: Der »Kammerdiener" (valet de chambre) wurde tübersetzt, während Begriffe wie »échanson« oder "sommelier« erhalten blieben, da »Mundschenk«, "Truchseß« oder "Kellermeister" zu sehr den mittelalterlichen Hof evozieren würden. Hinsichtlich der weiblichen Hofämter wurde nur die "dame d'honneur« zur »Ehrendame«, für alle anderen blieb die französische Bezeichnung erhalten, um die feinen Unterschiede zwischen "femme de chambre«, "fille de chambre«, "fille d'honneur« und »damoiselle» nicht zu verwischen ${ }^{53}$.

Hinsichtlich der Schreibweise von Eigennamen wurde wie folgt verfahren: Die Namen regierender Königinnen, Könige und Päpste wurden ins Deutsche ubertragen, also Katharina von Medici statt Caterina de' Medici oder Catherine des Médicis, alle anderen Namen wurden in ihrer ursprünglichen Form belassen: Anna d'Este statt Anna von Este, François de Lorraine statt Franz von Lothringen, Jacques de Savoie statt Jakob von Savoyen. Außerdem werden alle Personen, Männer wie Frauen, auch mit ihren Vornamen bezeichnet, ohne daß dadurch, wie es im historischen Roman oft geschieht, eine vermeintliche Nahe zwischen Leser oder Leserin und den geschichtlichen Figuren bezweckt wäre.

Bei Transkriptionen von ungedruckten Quellen wurde die Schreibweise beibehalten, Interpunktion und Akzentsetzung fur ein besseres Verstăndnis jedoch dem heutigem Gebrauch angepaßt. Abklirzungen innerhalb eines Satzes wurden aufgelöst, Titelbezeichnungen wie "Vostra Signoria Illustrissima" aber in ihrer Abkürzung belassen. Bei Drucken des 16. und 17. Jahrhunderts wurden bei Bedarf die Buchstaben »u« und »v« sowie »i« und »j« ausgetauscht, das »\&« und andere Kürzel aufgelöst. Offensichtliche Schreib- oder Druckfehler wurden stillschweigend korrigiert oder aber, sollte es sich um einen besonders aussagekräftigen Irrtum handeln, mit einer Anmerkung versehen. Das nach Annas Tod angefertigte Inventar ihres Hôtel de Nemours ist dem Anhang beigegeben und wird im Text mit dem Kürzel "Anhang A« zitiert. Fehlende Folio- oder Seitenangaben bedeuten, daß auch die entsprechende Quelle nicht paginiert ist.

${ }^{53}$ Zur Unterscheidung der weiblichen Hofämter: Anna-Manis MÖNSTER, Funktionen der dames et damoiselles d'honneur im Gefolge franzősischer Königinnen und Herzoginnen (14.-15. Jahrhundert), in: Jan HIRSCHBIEGEL, Werner PARAVICINI (Hg.), Das Frauenzimmer. Die Frau bei Hofe in Spătmittelalter und früher Neuzeit, Stuttgart 2000, S. 339-354, hier S. 342. 



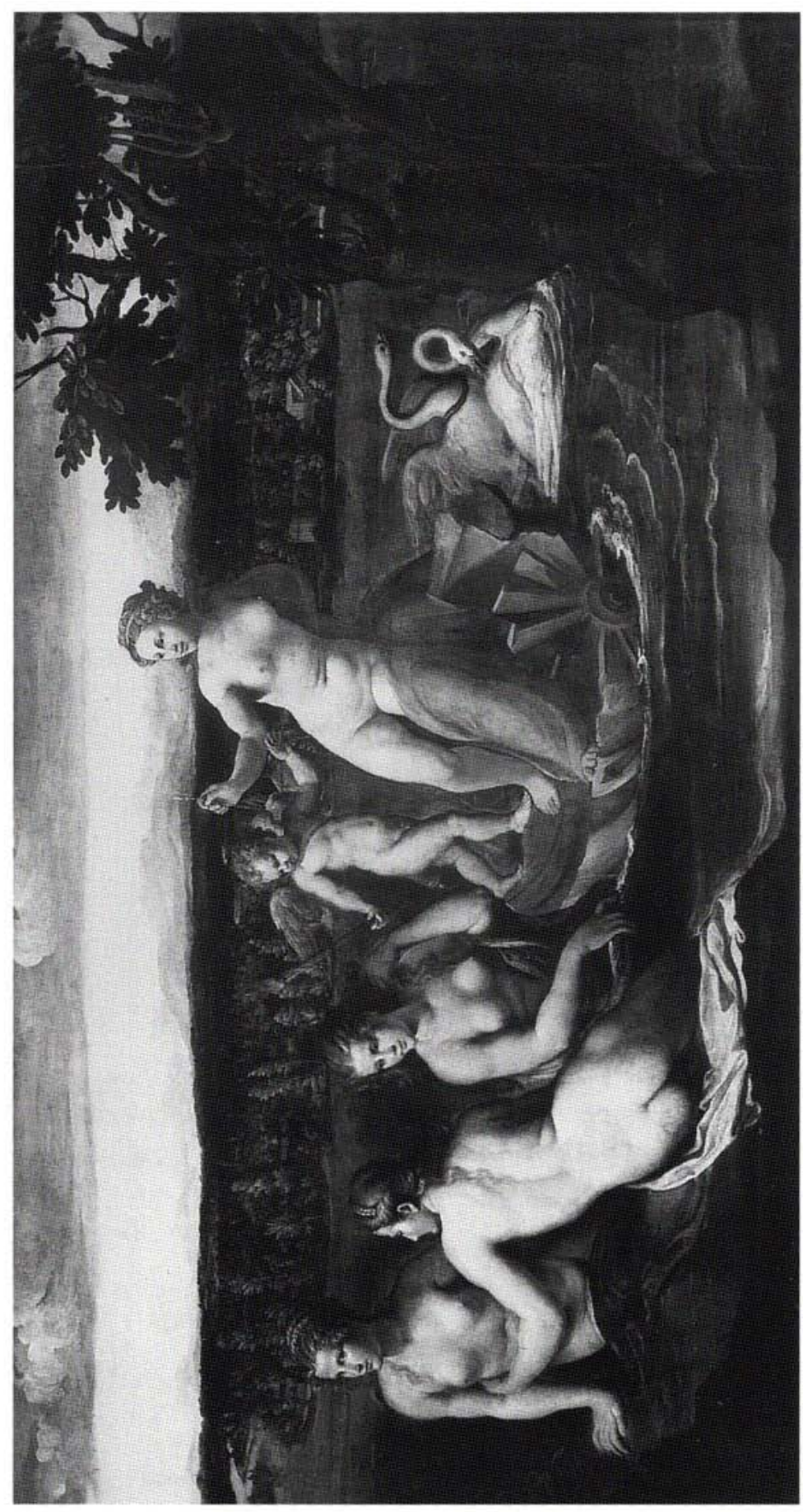

递 


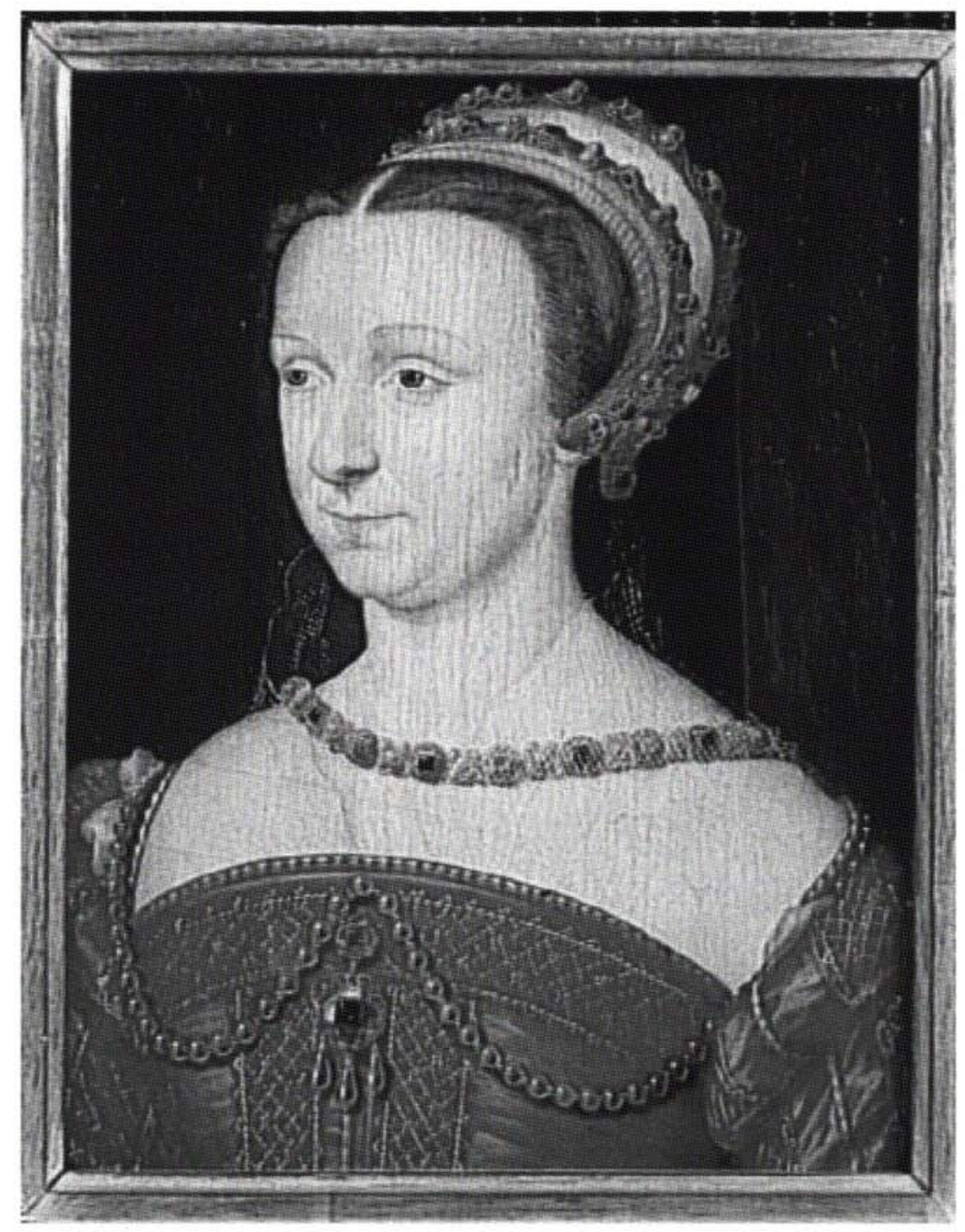

Abbildung 2 


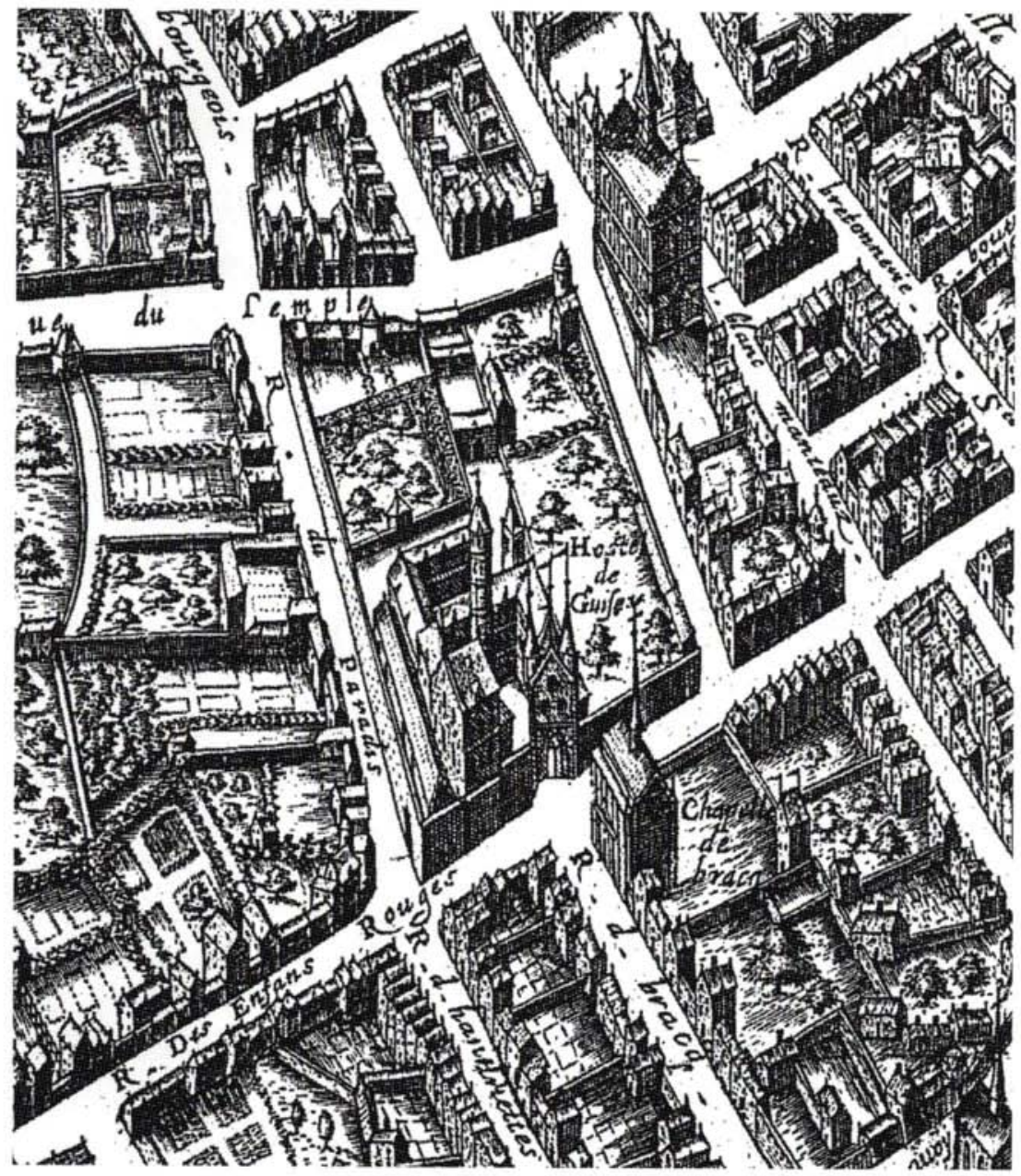




\section{INSTR $V M E N T V M D O$ Illuftrifs. E Eccelleniifs. D. Anna Eflei vxoris Illuftris/imi, Evotenti:fims Domini Ducis de Vmala.}

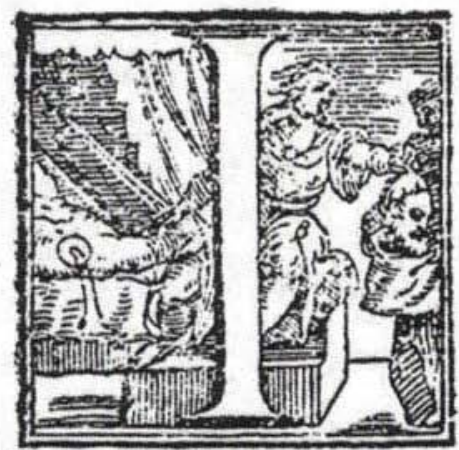

$N$ nomine, Amen. Anno einfdi tiuitatis millefimo quingentefi dragefimo outauo, indictione f vigefimo odauo menfis Septem ria, in palatio Reuerendifs \& ! Domini, D. Hipoliti Cardinalis Nunc habitationis Illuftrisfima reniffimę Domina. D. Renatę de Duciffx Ferrarix: fito in contra Fraucifi, \& un carnera eiufdé Sereniffrma Duciffæ refiden fentibus teltibns vocatis, \& rogatis, magnificis, \& clariffim confultis, domino Lanfrancho Gipfin de Lugn, ciue Ferı contrata fancti Leonardi, appellata Burgho Leonis, don lippo Thodo, ciue Ferrariz, de contrata Policini fanct omnibus Confiliarijs infrafcripui Illuftrifs. D. Ducis Ferr: 


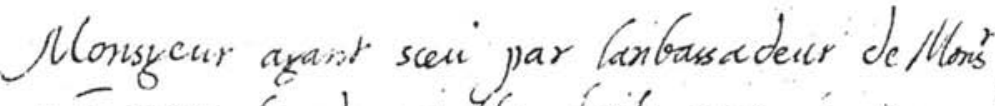

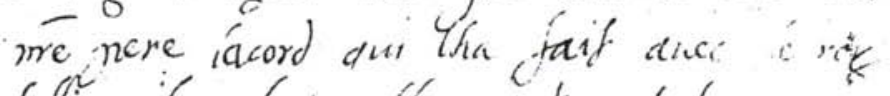

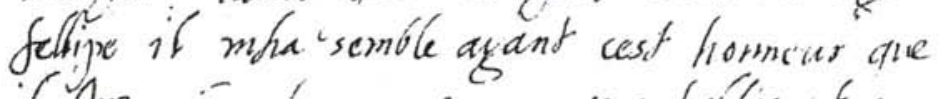

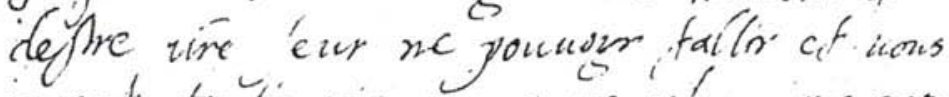

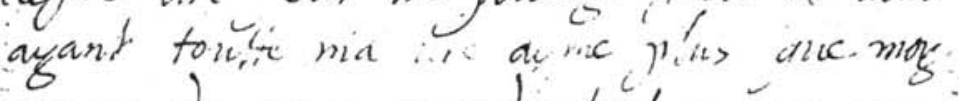

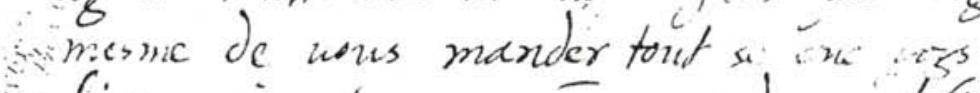

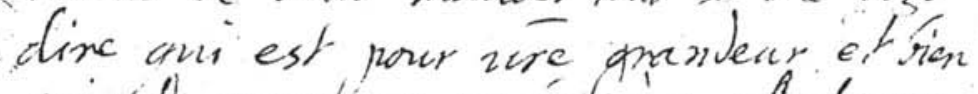

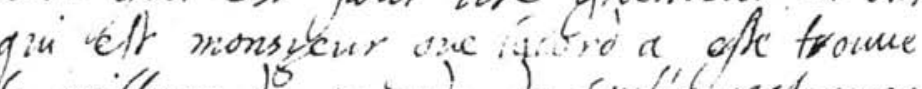

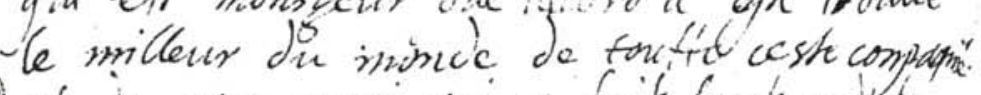

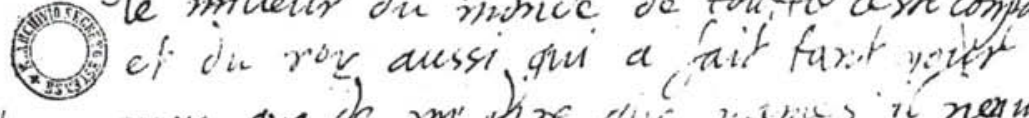

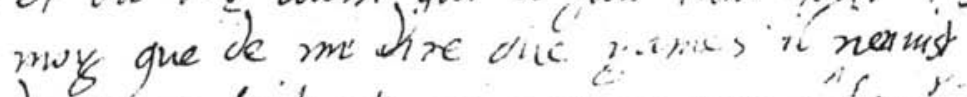

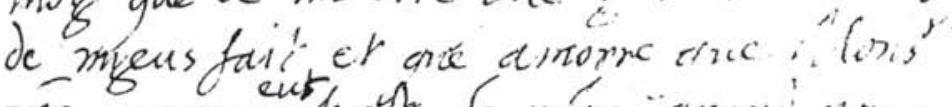

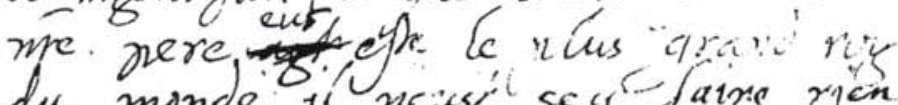
du monde il neixsit se is faine rion

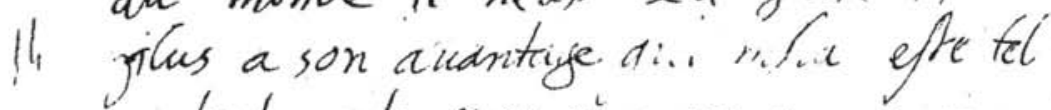
contantoment guenous poncs gacesses malys monszerer ge ne "me sarrogs garver de unous dire que, fort ie monde $x$ bínuce 6 plus estrange an est nosidie

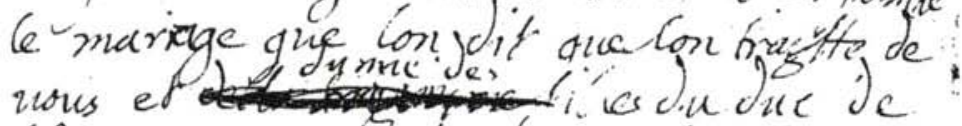

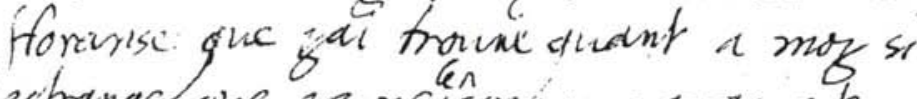

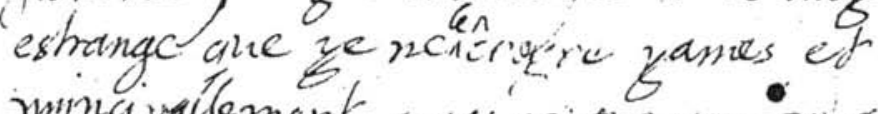

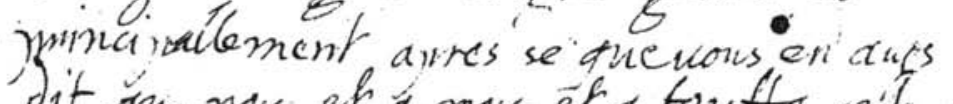

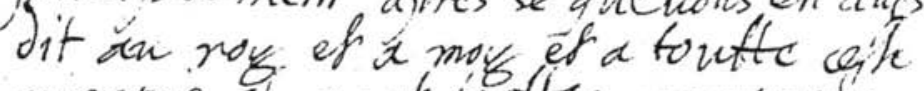
rongangmie of guarit touttes ses raglyoris 


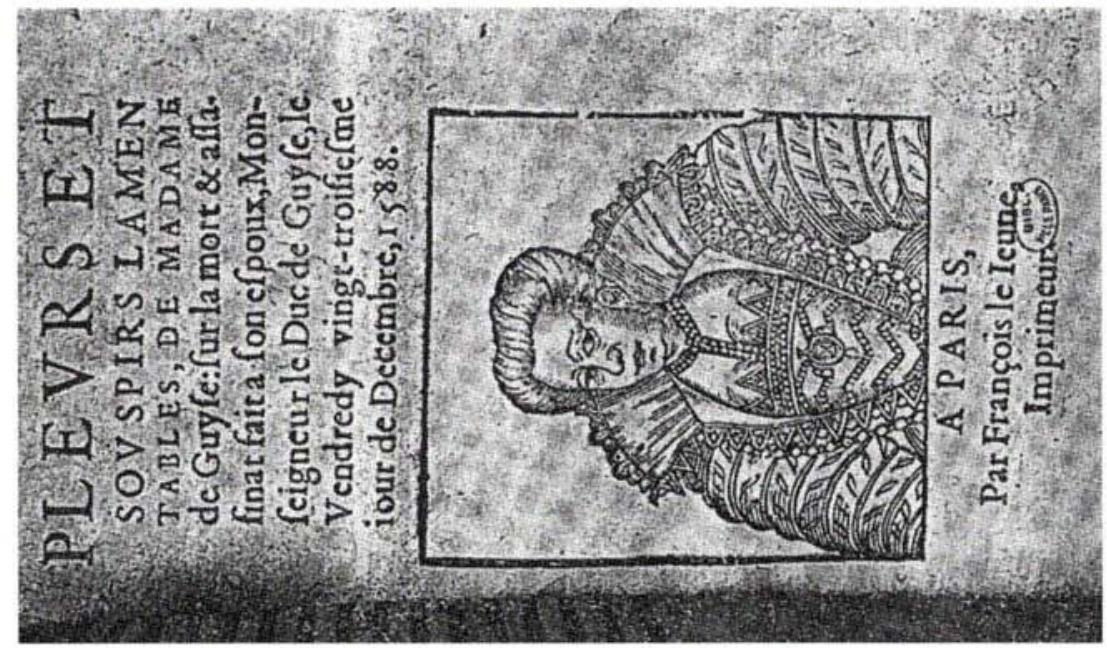

Abbildung 6

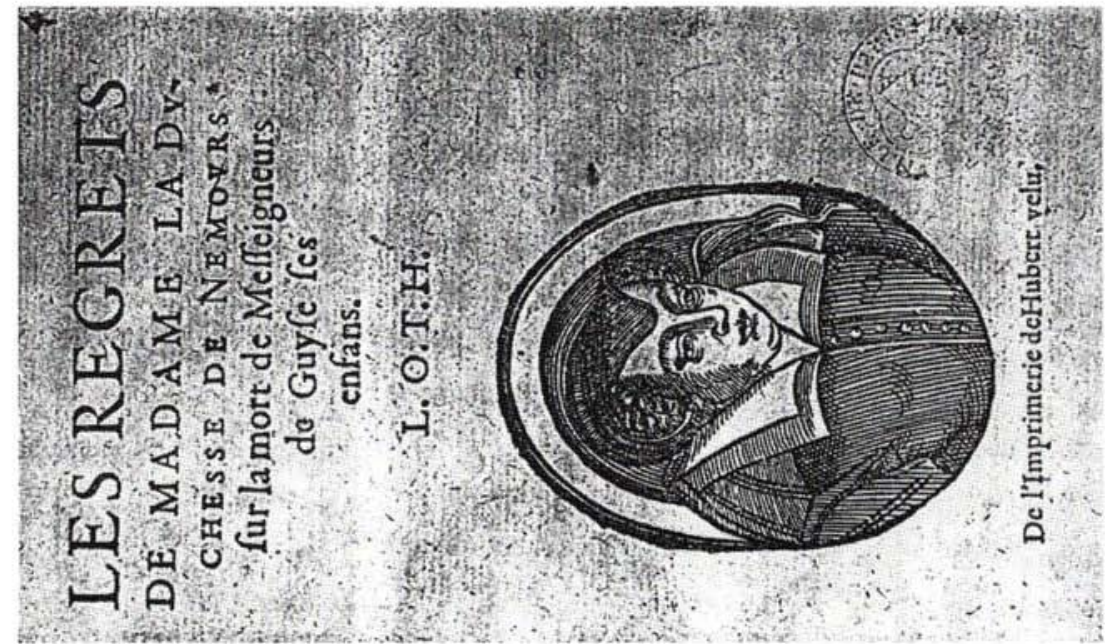

Abbildung 7 


\section{3}

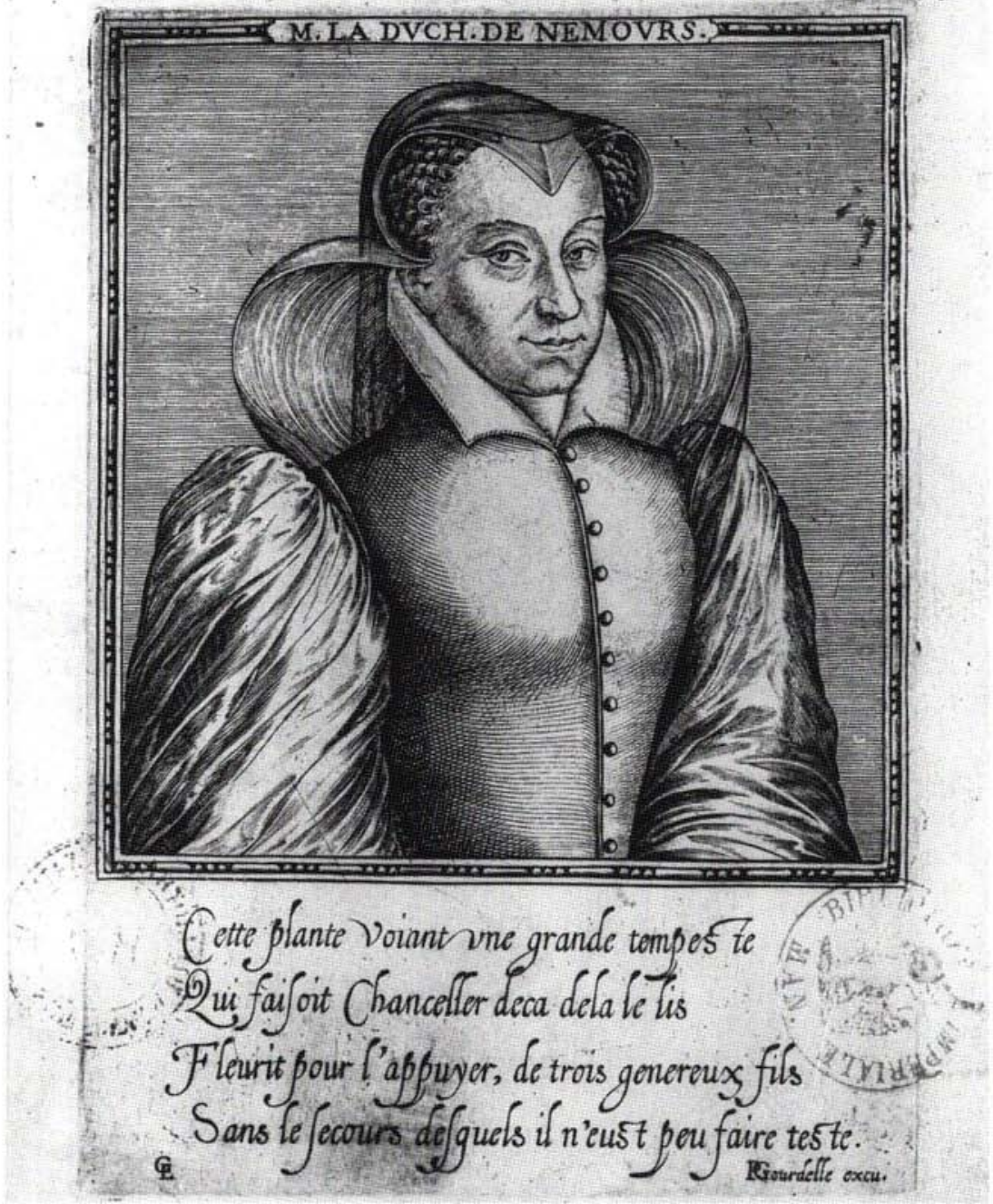




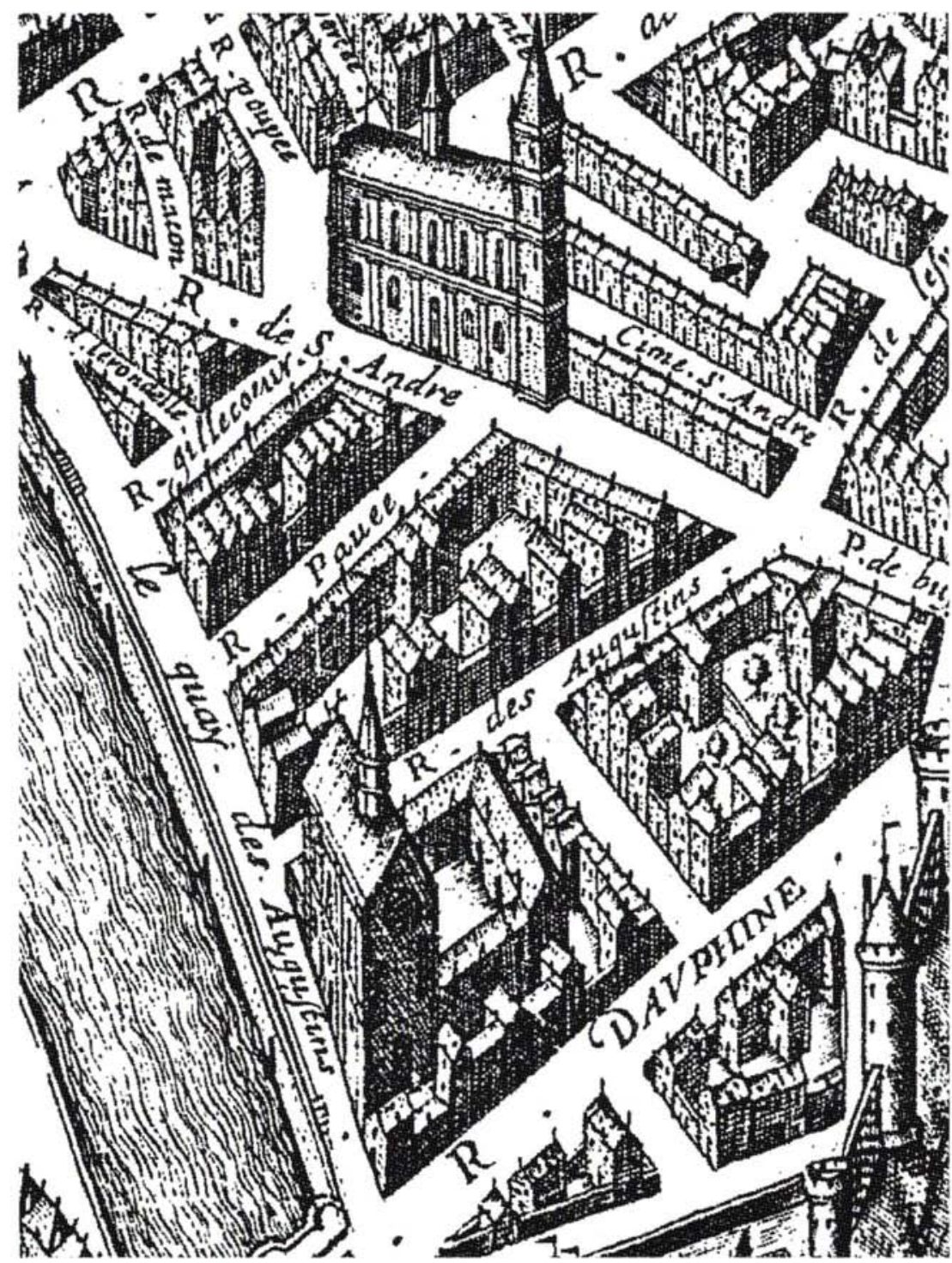

